\title{
CMAL: Cost-effective Multi-label Active Learning by Querying Subexamples
}

\author{
Guoxian Yu, Xia Chen, Carlotta Domeniconi, Jun Wang, Zhao Li, Zili Zhang, Xiangliang Zhang
}

\begin{abstract}
Multi-label active learning (MAL) aims to learn an accurate multi-label classifier by selecting which examples (or example-label pairs) will be annotated and reducing query effort. MAL is a more complicated and expensive process than single-label active learning, due to one example can be associated with a set of non-exclusive labels and the annotator has to scrutinize the whole example and label space to provide correct annotations. Instead of scrutinizing the whole example for annotation, we may just examine some of its subexamples with respect to a label for annotation. In this way, we can not only save the annotation cost but also speedup the annotation process. Given this observation, we introduce CMAL, a two-stage Cost-effective MAL strategy (CMAL) by querying subexamples. CMAL firstly selects the most informative example-label pairs by leveraging uncertainty, label correlation and label space sparsity. Specifically, the uncertainty of a label to an example can be reduced if its correlated labels already annotated to the example, and its uncertainty can be reduced also if more examples annotated to this label. Next, CMAL greedily queries the most probable positive subexample-label pairs of the selected example-label pair. In addition, we propose rCMAL to account for the representative of examples to more reliably select example-label pairs in the first stage. Extensive experiments on multi-label datasets from diverse domains show that our proposed CMAL and ICMAL can better save the query cost than stateof-the-art MAL methods. The contribution of leveraging label correlation, label sparsity and representative for saving cost is also confirmed.
\end{abstract}

Index Terms-Multi-label active learning, Multi-instance learning, label correlation, label sparsity, representative, uncertainty

\section{INTRODUCTION}

Label acquisition for the abundant unlabeled data is usually expensive because it requires the participation of human experts. Recent years have witnessed the proliferation and success of active learning (AL), which is widely used to improve the classification model by interacting with human experts. In AL, labels of selected examples, deemed as the most valuable, are iteratively queried, and used to update the classifier and improve the performance with

Manuscript received December 31, 2019; revised May 6, 2020; accepted June 5, 2020. This work is supported by Natural Science Foundation of China (61872300 and 61873214). Corresponding author: Jun Wang.

G. Yu and J. Wang are with the School of Software and Joint SDU-NTU Centre for Artificial Intelligence Research, Shandong University, Jinan 250101, China (e-mail: guoxian85@gmail.com,kingjun@sdu.edu.cn).

$X$. Chen and Z. Li are with the Alibaba Group, Hangzhou 310000, China. (e-mail: $\{x i a . c x, l i z h a o . l z\} @ a l i b a b a . c o m)$

C. Domeniconi is with the Department of Computer Science, George Mason University, VA 22030, USA (e-mail: carlotta@cs.gmu.edu)

Z. Zhang is with the College of Computer and Information Science, Southwest University, Chongqing 400715, China (e-mail: zhangzl@swu.edu.cn).

$X$. Zhang is with the King Abdullah University of Science and Technology,

Thuwal, SA (e-mail: xiangliang.zhang@kaust.edu.sa) reduced cost [1], [2]. The canonical setting in $\mathrm{AL}$ involves with single-label examples, i.e., examples with exactly one label. AL under traditional single-label setting studies the example-based selection criteria to iteratively select the most valuable examples and then queries their labels from the oracle (i.e., human annotators) [1]. Multi-label scenarios, where examples can have more than one label, are more challenging. This is because the process of querying the multi-label examples needs to examine the whole label space, even if the relevant labels are sparse. Early multi-label active learning (MAL) algorithms adhere to example-based selection strategies typically-used in single-label setting, and simultaneously query the relevance of the selected example with respect to all labels in the label space [3]-[7]. These MAL algorithms pose a risk of wasting cost and time, when querying an example with a large label space, since the oracle has to make a tedious scrutiny of selected examples with respect to each label of the whole label space. More recent MAL approaches advocate to select examplelabel pairs, and then query the relevance between the example and label $[\overline{8}]-[13]$. Empirical study has attested that the query cost can be much reduced by an appropriate example-label pair selection strategy.

The relevance between an example and a label depends on the patterns of the example itself [14], [15]. For example, a natural image often includes different objects (subexamples), the semantic tags of the image are often triggered by its salient subexamples. However, almost all AL approaches ask the oracle to scrutinize the whole image (all subexamples) and then annotate the semantic tags. But in fact, the image can be more easily annotated by just asking the oracle to annotate some salient subexamples, instead of the whole image. From this perspective, querying the examplelabel pair may result in information redundancy and in a waste of resources (or unnecessary repeated queries), if its relevant subexamples to the label can be queried as early as possible. An example of this observation is illustrated in Figure 11. From Figure 11, we can clearly see that the annotator can more easily annotate the relevance of example $\mathbf{X}_{i}$ with respect to label ' 1 ', by only annotating its specific subexamples $\left(\mathbf{x}_{4}\right.$ or $\left.\mathbf{x}_{5}\right)$, instead of all of its subexamples $\left(\mathbf{x}_{1}, \mathbf{x}_{2}, \mathbf{x}_{3}, \mathbf{x}_{4}\right.$ and $\left.\mathbf{x}_{5}\right)$. Thus, we can further reduce the query cost of MAL by querying the most informative and plausible positive subexample-label pairs, instead of the canonically studied example-label pair.

Informativeness and representative are the two widely used criteria to select the most valuable examples for query in AL [9], [16]. Informativeness prefers the examples containing more information that the current classification model doesn't know, while representative prefers the examples that can well represent 
the overall input patterns of unlabeled data. Empirical studies have shown that combining the two criteria helps to make more effective selection strategies [10]. In addition, it's advised that both the label correlation and label space sparsity are important for multi-label learning $[17]-[19]$. For MAL, the label correlation can be used to reduce the uncertainty between an example-label pair given its correlated labels already annotated to the example. Label space sparsity can be used to reduce too excessive query for an example and a label, since a multi-label example is generally annotated with a small subset of the whole labels and each label is often relevant to a small portion of examples.

Motivated by these observations, we introduce a two-stage Cost-effective MAL (CMAL) framework. In each iteration, CMAL first makes use of uncertainty, label correlation and label space sparsity to select the most informative example-label pair, and then surrogates the example-label relevance query by greedily querying its most likely positive subexample-label pairs. Thus, CMAL is expected to reduce the query cost by querying subexample-label pairs as few as possible. The main idea of CMAL is illustrated in Figure 1. We simply assume that the cost (e.g., effort) of querying an example-label pair is larger than that of querying its subexample-label pair. In addition, we further develop a new criterion to quantify the representative of example-label pair and introduce representative-based CMAL (rCAML), which selects both informative and representative example-label pairs for the follow-up subexample-label query.

This paper is a significant extension to our previous work [20], where as a showcase we have demonstrated the efficacy of subexample-label query for MAL. We move forward to more extensive related works, deep analysis for subexample-based MAL, a new representative-based CMAL (rCMAL), more comparing methods and datasets for the experimental study, and more comprehensive ablation study and analysis. The main contributions of this paper are:

(1) We observe that the cost of querying an example-label pair can be reduced by querying its subexample-label pairs, and advocate to perform active learning on examples by querying the subexamples of the example.

(2) We propose two cost-effective multi-label active learning algorithms (CMAL and rCMAL). CMAL introduces a weighted uncertainty measure based on label correlation and label space sparsity to select the most informative example-label pairs, and then surrogates the query of target example-label pair by querying its most likely positive subexample-label pairs. rCMAL extends CMAL by designing a novel strategy to select both informative and representative example-label pairs. The proposed query strategies of CMAL and rCMAL are different from existing active learning strategies for multi-label data and for multi-instance data [6], [10], [21]-[23].

(3) Experimental results on publicly available datasets show that CMAL and rCMAL can achieve a better (comparable) performance than state-of-the-art methods at a same (lower) cost [6], [10], [21]-[23], and rCMAL achieves the best performance in most cases.

We organize the rest of this paper as follows. In Section 2 we introduce the related works on MAL. The proposed CMAL and rCMAL algorithms are presented in Section 3 Experimental results and analysis are presented and discussed in Section 4 followed with conclusions and discussions in Section 5 .

\section{Related Work}

The key task of AL is to design the selection criteria of examples or example-label pairs for query. These criteria can be roughly divided into three categories: informativeness based criteria (e.g., uncertainty-based criteria [5], [9], [13], [24]-[26], mutual information based criteria [27] and expected error reduction based criteria [28]-[30]); representative-based criteria (e.g., clustering based criteria [16], [31], [32], diversity-based criteria [9] and density based criteria [33]); the criteria combining informativeness and representative [9], [10], [34]-[37]. Informativeness measures the ability of an example in reducing the uncertainty of a classification model and in improving the model's generalization ability, whereas representative measures whether an example well represents the overall input patterns of unlabeled data and prevents model bias [10]. Li et al. [5] first proposed a max-margin prediction uncertainty strategy and a label cardinality inconsistency strategy, and then integrated them into an adaptive framework to perform MAL by iteratively querying the most valuable examples. To deal with the problem of positive label sparsity in multi-label learning and to achieve a near-optimal MAL algorithm, Vasisht et al. [27] proposed a novel example selection strategy using an approximation to the mutual information based on the sparse Bayesian multilabel classification model [38]. Qi et al. [8] introduced a twodimensional MAL algorithm to query example-label pairs by minimizing the multi-label Bayesian classification error bound. To handle the outlier labels and to jointly leverage informativeness and representative of examples to perform more effective MAL, Du et al. 34 derived a robust MAL algorithm based on maximum corr-entropy criterion by merging uncertainty and representative. Huang et al. [10] injected all labeled examples and unlabeled examples into a margin-based AL method from the viewpoint of min-max to query single-label examples that are both informative and representative, and extend it to multi-label setting to query example-label pairs by incorporating the correlation among labels.

However, these MAL methods mostly focus on designing effective selection strategies by measuring the informativeness of examples or example-label pairs. In recent years, some efforts moved toward investigating more practical AL methods. Some approaches consider the case that the examples can be annotated with different annotators with diverse quality and costs, and try to select the example-annotator pairs [12], [39]-[41]. Different from typical active learning methods that select low confident example for query, some methods follows the self-paced learning [42] and greedily assign the high confidently predicted pseudo-labels to unlabeled training examples without human oracle [43]. Huang et al. [44] introduced a novel query strategy that is able to collect richer information while requires less expertise from the annotator. Particularly, instead of querying the example or example-label pairs, this strategy queries the relevance ordering of target label pairs with respect to a selected example. Unlike these works, we investigate the cost-effective MAL based on the cost margin between querying an example-label pair and its subexample-label pairs.

From the perspective of querying the relevance of subexamplelabel pairs, our work has a close connection with multi-instance learning (MIL), where each example can be viewed as a bag that is made of multiple subexamples (a.k.a. instances) [45], [46]. In MIL, a bag is positive for a label if at least one of its instances is annotated with this label, while the bag is negative for a label if all its instances are not annotated with this label. Compared with the well-studied single-instance active learning, only few 
efforts have been made toward the more complex multi-instance active learning task, and our solutions have distinctions from all of them. Some active MIL approaches assume the training bags are either positive or negative, and focused on determining the labels of unlabeled instances of the positive bags to train an instancelevel classifier [21] or bag-level one [47]. On the contrary, our CMAL and rCMAL aims to query the labels of unlabeled bags (i.e., examples) for an accurate bag-level predictor. Some other methods [48], [49] also aim at querying the labels of unlabeled bags to make a bag-level predictor, but they have to scrutinize all instances of the bag before annotating it, or only execute one round of query and permit the oracle to annotate a group most valuable instances [50]. Furthermore, these multi-instance active learning solutions are designed for binary case, which is a degenerated case of the multi-label classification problem investigated here. Retz et al. [22] and Huang et al. [23] recently studied MAL with multiinstances, they either have to simultaneously query the relevance of the selected bag with respect to all labels, or the relevance of the selected bag-label pair based on all its instances. In contrast, our proposed CMAL and rCMAL query the relevance of the selected bag-label pair by querying its most plausible positive instance-label pairs as fewer as possible, and thus has a significantly reduced query cost.

\section{The Proposed method}

\subsection{Problem Statement}

Without loss of generality, suppose $\mathcal{L}=\left\{\left(\mathbf{X}_{i}, \mathbf{Y}_{i}\right)\right\}_{i=1}^{l}$ is the initial and small training set with $l$ labeled examples, where $\mathbf{X}_{i} \in$ $\mathbb{R}^{d}$ is the feature vector for the $i$-th example, and $\mathbf{Y}_{i} \in\{-1,+1\}^{q}$ represents the labels of the $i$-th example in the $q$-dimensional label space. If $\mathbf{X}_{i}$ is annotated with the $c$-th label, $\mathbf{Y}_{i c}=1$; otherwise $\mathbf{Y}_{i c}=-1$. Let $\mathcal{U}=\left\{\mathbf{X}_{i}\right\}_{i=l+1}^{l+u}$ be a large pool of $u(l \ll u)$ unlabeled examples, $n=l+u$. Let $\mathcal{B}_{i}=\left\{\mathbf{x}_{i 1}, \mathbf{x}_{i 2}, \cdots, \mathbf{x}_{i n_{i}}\right\}$ represent the subexamples of $\mathbf{X}_{i}$, and $n_{i}=\left|\mathcal{B}_{i}\right|$ is the number of subexamples of $\mathbf{X}_{i}$. Suppose each subexample $\mathbf{x}_{i k} \in \mathbb{R}^{d^{\prime}}$ is associated with labels $\mathbf{y}_{i k} \in\{-1,+1\}^{q}$. Similarly, if $\mathbf{x}_{i k}$ is associated with the $c$-th label, $\mathbf{y}_{i k c}=+1$; otherwise $\mathbf{y}_{i k c}=-1$. $\mathcal{L}_{\text {sub }}$ encodes the set of training subexamples from $\mathcal{L}$, and $\mathcal{U}_{\text {sub }}$ represents the unlabeled subexamples from $\mathcal{U}$. From the MIL perspective [45], [46], [51], the complex example can also be modeled as a bag and its subexamples can be viewed as instances of that bag. We obey the canonical MIL principle that an example (or bag) is positive for a label if it has at least one subexamples (or instances) annotated with this label, while an example is negative for a label if all its subexamples are not annotated with this label. Thus, although all the examples in $\mathcal{L}$ are labeled, the labels of subexamples in $\mathcal{L}_{\text {sub }}$ from positive examples are unknown. MAL aims to improve the learning performance on multi-label examples, by querying the most valuable example-label pairs.

Our work is motivated by the observation that querying the relevance of a subexample-label pair of a selected example-label pair is more easier, as illustrated in Figure 1. Thus, we can further reduce the query cost of MAL by querying the most plausible positive subexample-label pairs, instead of the canonically studied example-label pair or all the subexample-label pairs. The labels of an example are driven by the labels of its subexamples, so the query of example-label pair can be alternatively made by querying its subexample-label pairs with a lower cost.

Given this observation, the selection strategy can be divided into two stages, selecting the example-label pair, and then choosing the most plausible positive subexample-label pair of the selected example-label pair. To concrete this idea, we need to train an example-level classifier for selecting informative example-label pair and a subexample-level classifier to choose the potential positive subexample-label pairs. An intuitive solution for example-level classifier is to transform a multi-label classification problem into $q$ independent binary classification problems (one per label) via the "one-vs-all" scheme. Because of the simplicity and effectiveness of Support Vector Machine (SVM) classifiers for binary classification, in this work, we conduct multi-label classification under such a scheme using an SVM for each label. As to the subexample-level classifier, for a particular label, all subexamples from its negative examples are known as negative, while the relevant subexamples from its positive examples are unknown. Thus, the training subexamples from $\mathcal{L}_{s u b}$ are not all labeled, and SVM cannot be used for subexample classification. A multi-instance SVM (miSVM) [52] is a binary classifier that deals with the multiple instance learning problem without knowing the subexample-level labels, and it aims to induce a subexample-level classifier with labels of bags. Thus, for subexample classification, we adopt miSVMs for the $q$ independent binary classification problems. Suppose $f_{1}, f_{2}, \cdots, f_{q}$ are $q$ SVM classifiers, and $g_{1}, g_{2}, \cdots, g_{q}$ are $q$ miSVM classifiers for examples and subexamples, respectively. Each classifier corresponds to a particular label. Let $\hat{\mathbf{Y}}_{i c}=\operatorname{sign}\left(f_{c}\left(\mathbf{X}_{i}\right)\right)$ and $\hat{\mathbf{y}}_{i k c}=\operatorname{sign}\left(g_{c}\left(\mathbf{x}_{i k}\right)\right)$ be the predictions of example $\mathbf{X}_{i}$ and subexample $\mathbf{x}_{i k}$ with repsect to the $c$-th label, respectively. We elaborate on the proposed CMAL and $\mathrm{rCMAL}$ in the following subsections.

\subsection{Cost-effective Multi-label Active Learning (CMAL)}

In this subsection, we introduce the proposed Cost-effective Multi-label Active Learning algorithm, called CMAL. Inspired by the observation that the cost for querying a subexample-label pair is smaller than its example-label pair, we expect to reduce the annotating cost for an example-label pair by querying its subexample-label pairs as few as possible. At each iteration of MAL, CMAL first selects the most informative example-label pair based on prediction uncertainty, which is further weighted by label correlation and label space sparsity. Next, it chooses the most likely positive subexample-label pair, among all subexamplelabel pairs of the selected example-label pair, and then queries the relevance of the selected subexample-label pair. In the following, we describe the selection strategies for example-label pairs and subexample-label pairs, respectively.

\subsubsection{Select the Most Informative Example-Label Pair}

To choose the most informative example-label pair, we adopt the widely-used uncertainty criterion, which quantifies the confidence of the current predictor in classifying the example. A lower confidence corresponds to a larger uncertainty value and means the selected example-label pair having a larger informativeness to improve the classifier [1]. In other words, if the classifier is uncertain about the prediction made on an example, then knowing the labels of the example may be helpful in improving the classifier, because it provides information that the current classifier does not have yet. Since we adopt SVM for predicting the label of bags, we can approximate the uncertainty of an example-label pair based on the distance between the example and the decision boundary. Based on the maximum margin principle, a smaller distance suggests a larger uncertainty. The decision boundary with respect to the $c$-th 


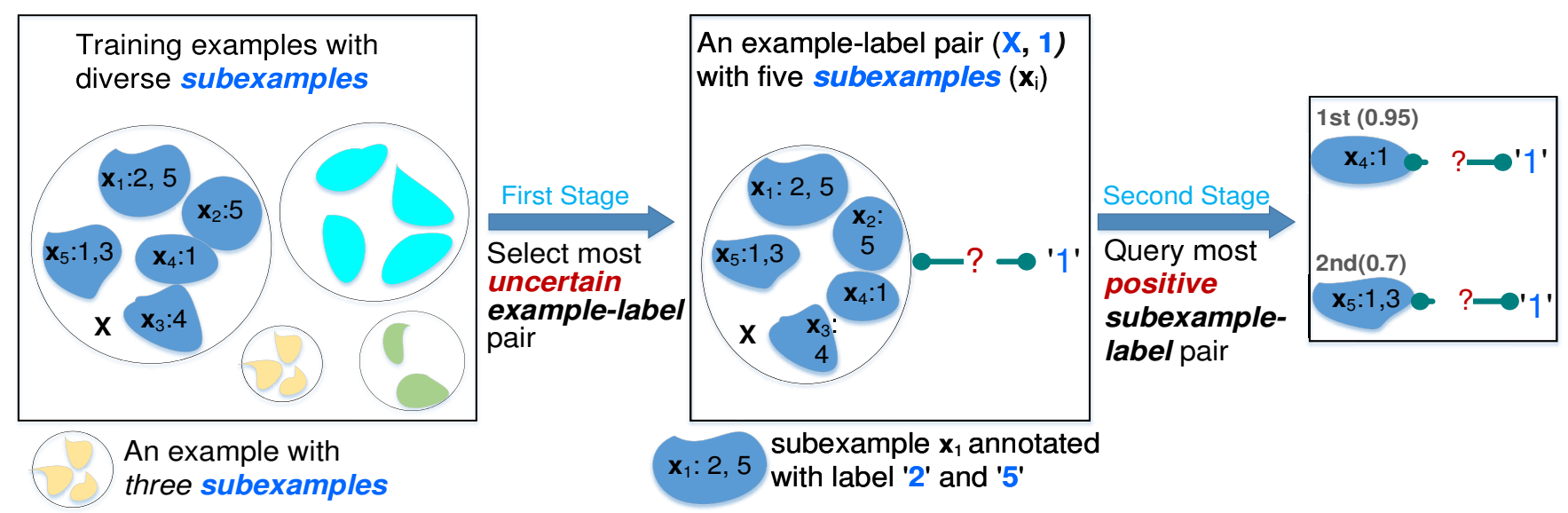

Fig. 1. An illustration of the main idea of CMAL, $\mathbf{X}$ is a complex example (bag) composed with five subexamples (instances), which are relevant with different labels of this multi-label example. CMAL first selects the most uncertain example-label pair ( $\mathbf{X}$ w.r.t. label '1'), and then queries the most likely positive subexample-label pairs ( $\mathbf{x}_{4}$ or $\mathbf{x}_{5}$ w.r.t. ' 1 ') of the selected example-label pair. The annotator can more easily annotate the example by checking its subexamples (i.e., $\mathbf{x}_{4}$ and $\mathbf{x}_{5}$ ), instead of scrutinizing the whole example (or all subexamples).

label in the example-level can be defined as $f_{c}(\mathbf{X})=0$, and the distance between $\mathbf{X}_{i}$ and that label is $\left|f_{c}\left(\mathbf{X}_{i}\right)\right|$. This distance can be transformed into a measure of uncertainty as:

$$
U\left(\mathbf{X}_{i}, c\right)=\frac{1}{\left|f_{c}\left(\mathbf{X}_{i}\right)\right|}
$$

Unlike single-label learning that each example is exclusively associated with only one label, in multi-label learning, an example can be associated with a set of non-exclusive labels. As such, it is important to make use of label correlation for effective multilabel learning [17], [18]. However, the binary SVM does not explicitly account for the label correlations. Based on the label correlation, the information embedded in a unknown label of an example can be supplemented from its correlated labels that are relevant for the same example. In this way, we can reduce the number of queries and save cost of MAL by properly using label correlation. It is recognized that multi-label datasets often have the charateristics of label space sparsity. Namely, only a small fraction of examples are relevant for a given label and each example is annotated with a small subset of the whole labels. From these analysis, we incorporate label correlation and label space sparsity to define a weighted uncertainty based example-label pair selection strategy. For this purpose, we estimate the pairwise label correlation matrix $\mathbf{W}$ as follows:

$$
\mathbf{W}\left(c_{1}, c_{2}\right)=\frac{\sum_{i=1}^{l+u}\left[\mathbf{Y}_{i c_{1}}=+1, \mathbf{Y}_{i c_{2}}=+1\right]}{\sum_{i=1}^{l+u}\left[\mathbf{Y}_{i c_{1}}=+1 \text { or } \mathbf{Y}_{i c_{1}}=-1, \mathbf{Y}_{i c_{2}}=+1\right]}
$$

where $[x]=1$ if $x$ is true, $[x]=0$ otherwise. $\mathbf{W} \in \mathbb{R}^{q \times q}$ measures the empirical conditional probability that an example is annotated with label $c_{1}$, given that the example was already annotated with label $c_{2}$. This label correlation is widely adopted in multi-label learning for its simplicity and intuitiveness [20], [53]. Other estimations of label correlation can also be adopted [17]. Note, $\mathbf{W}$ is iteratively updated as more queried labels to mitigate the bias, since $\mathbf{W}\left(c_{1}, c_{2}\right)$ is based on only the examples already annotated with $c_{1}$ or $c_{2}$.

Based the estimated label correlation, we define two weights as follows:

$$
w_{1}\left(\mathbf{X}_{i}, c\right)=1-\frac{\sum_{c_{1} \in \mathcal{Y}+\left(\mathbf{X}_{i}\right)} \mathbf{W}\left(c, c_{1}\right)}{q}, w_{2}(c)=1-\frac{n_{c}^{+}}{n}
$$

Here $w_{1}\left(\mathbf{X}_{i}, c\right) \in[0,1]$ is the weight of the example-label pair $\left(\mathbf{X}_{i}, c\right)$, and $w_{2}(c) \in[0,1]$ is the weight of the $c$-th label. $\mathcal{Y}^{+}\left(\mathbf{X}_{i}\right)$ is the set of queried relevant labels of $\mathbf{X}_{i}$ and $n_{c}^{+}$is the number of examples annotated with label $c . w_{1}\left(\mathbf{X}_{i}, c\right)$ is driven by observation that if $c$ is highly correlated with $c_{1}$ (a large $\left.\mathbf{W}\left(c, c_{1}\right)\right)$, then $c$ is also possibly annotated to $\mathbf{X}_{i}$, given $c_{1}$ is already annotated to $\mathbf{X}_{i}$. As a result, the larger the correlation between $c$ and labels annotated to $\mathbf{X}_{i}$ is, the less uncertain $\left(\mathbf{X}_{i}, c\right)$ is. In addition, the larger the number of queried labels for an example is, the less uncertain the example is. $w_{2}(c)$ is driven by the fact that the more examples annotated with $c$, the less uncertain $c$ is.

To this end, we quantify the updated informativeness of an example-label pair as follows:

$$
\tilde{U}\left(\mathbf{X}_{i}, c\right)=w_{1}\left(\mathbf{X}_{i}, c\right) * w_{2}(c) * U\left(\mathbf{X}_{i}, c\right)
$$

Then we can select the most informative example-label pair $\left(\mathbf{X}_{i^{*}}, c^{*}\right)$ for query as follows:

$$
\left(\mathbf{X}_{i^{*}}, c^{*}\right)=\arg \max _{\mathbf{X}_{i} \in \mathcal{U}, c \in \mathcal{Q}_{l}\left(\mathbf{X}_{i}\right)} \tilde{U}\left(\mathbf{X}_{i}, c\right)
$$

where $i^{*}$ and $c^{*}$ are the selected example and label indices, respectively, $\mathcal{Q}_{l}\left(\mathbf{X}_{i}\right)$ is the set of un-queried labels for $\mathbf{X}_{i}$.

\subsubsection{Select and Query Subexample-label Pair}

Given the selected example-label pair $\left(\mathbf{X}_{i^{*}}, c^{*}\right)$, we try to minimize the cost for querying its relevance as much as possible. Because the cost for querying a subexample-label pair is smaller than its example-label pair, a surrogate idea is to query its most likely positive subexample-label pair. If the query result is positive, the classifier obtains direct evidence for positive relevance between the queried example and label. If the query result is negative, the classifier knows that the selected example is likely to be negative for the label, and this information reduces the example ambiguity.

Hence, CMAL then selects the most likely positive subexamplelabel pair of the selected example-label pair $\left(\mathbf{X}_{i^{*}}, c^{*}\right)$ and queries its relevance. The selection strategy is formalised as follows:

$$
\left(\mathbf{x}_{i^{*} k^{*}}, c^{*}\right)=\arg \max _{\mathbf{x}_{i^{*} k} \in \mathcal{Q}_{s u b}\left(\mathcal{B}_{i^{*}}\right)} g_{c^{*}}\left(\mathbf{x}_{i^{*} k}\right)
$$

where $k^{*}$ is the selected subexample index of example $\mathbf{X}_{i^{*}}$ and $\mathcal{Q}_{\text {sub }}\left(\mathcal{B}_{i^{*}}\right)$ denotes the set of un-queried subexamples of the example-label pair $\left(\mathbf{X}_{i^{*}}, c^{*}\right)$. 
In each iteration, after querying the subexample-label pair $\left(\mathbf{x}_{i^{*} k^{*}}, c^{*}\right)$, the answer is either positive (relevant, +1$)$ or negative (irrelevant, -1). Follow the standard MIL assumption, a bag is positive with respect to a particular label if at least one of its instances is positive, while the example is negative if all its instances are negative for that label [45], [46], [51]. Therefore, we formulate the following update strategy for the query of subexample-label pairs.

If the feedback is positive, then the relevance between the selected example and label is positive, and CMAL performs the following updates:

(i) $\left(\mathbf{X}_{i^{*}}, c^{*}\right)$ is annotated with +1 , removed from the unlabeled example set $\mathcal{U}$ and added to the labeled example set $\mathcal{L}$.

(ii) $\left(\mathbf{x}_{i^{*} k^{*}}, c^{*}\right)$ is annotated with +1 , removed from the unlabeled subexample set $\mathcal{U}_{s u b}$ and added to the training subexample set $\mathcal{L}_{\text {sub }}$.

(iii) The current two classification models $f_{c^{*}}(\mathbf{X})$ and $g_{c^{*}}(\mathbf{x})$ are updated with the new $\mathcal{L}$ and $\mathcal{L}_{\text {sub }}$, respectively.

On the other hand, if the feedback is negative, we cannot simply determine $\left(\mathbf{X}_{i^{*}}, c^{*}\right)$ as negative, since there may exist other positive subexample-label pairs of $\left(\mathbf{X}_{i^{*}}, c^{*}\right)$. The example-label pair $\left(\mathbf{X}_{i^{*}}, c^{*}\right)$ is negative if all its subexample-label pairs have been queried and in each case the feedback was negative. So for a negative feedback, CMAL does the following updates:

(i) If the subexample-label pair $\left(\mathbf{x}_{i^{*} k^{*}}, c^{*}\right)$ is the last being queried, and in each case the feedback was negative, then $\left(\mathbf{X}_{i^{*}}, c^{*}\right)$ is annotated with -1 , removed from $\mathcal{U}$ and added to $\mathcal{L}$.

(ii) $\left(\mathbf{x}_{i^{*} k^{*}}, c^{*}\right)$ is annotated with -1 , removed from $\mathcal{U}_{s u b}$ and added to $\mathcal{L}_{\text {sub }}$.

(iii) $g_{c^{*}}(\mathbf{x})$ is updated based on the new $\mathcal{L}_{s u b}$. If (i) is executed, then $f_{c^{*}}(\mathbf{X})$ is updated based on the new $\mathcal{L}$.

The pseudo-code of CMAL is summarized in Algorithm 1 CMAL iteratively selects the most informative example-label pair (line 3-6) and then iterative queries the most likely positive subexample-label pair of the selected example-label pair (line 7-11). If the obtained feedback for the selected subexample-label pair is positive, then the selected example-label pair is also positive (line 13-15). Otherwise, if all subexample-label pairs of the selected example-label pair are negative, then the selected example-label pair is deemed as the negative (line 16-18). After that, both the bag-level predictor $f_{c^{*}}(\mathbf{X})$ and instance-level predictor $g_{c^{*}}(\mathbf{x})$ are updated based on augmented training examples and subexamples (line 19). These update rules are based on the observation that a positive example-label pair has at least one positive subexamplelabel pair. If the selected example-label pair is positive, the oracle will meet the positive subexample-label pair early in the query process, which avoids further querying other subexamples and thus saves the cost. If the selected example-label pair is negative, the learner can uncover the negative relationship by iteratively querying its all subexample-label pairs. Other query strategies for subexample-label pairs of the selected negative example-label pair will be studied in Section 4.5

\subsection{Representative-induced Cost-effective Multi-label Active Learning (rCMAL)}

It is recognized that besides the informativeness, the representative of samples is also helpful for AL [9], [34], [35]. Given that, we further introduce a representative-induced Cost-effective Multilabel Active Learning algorithm called rCMAL. Particularly,

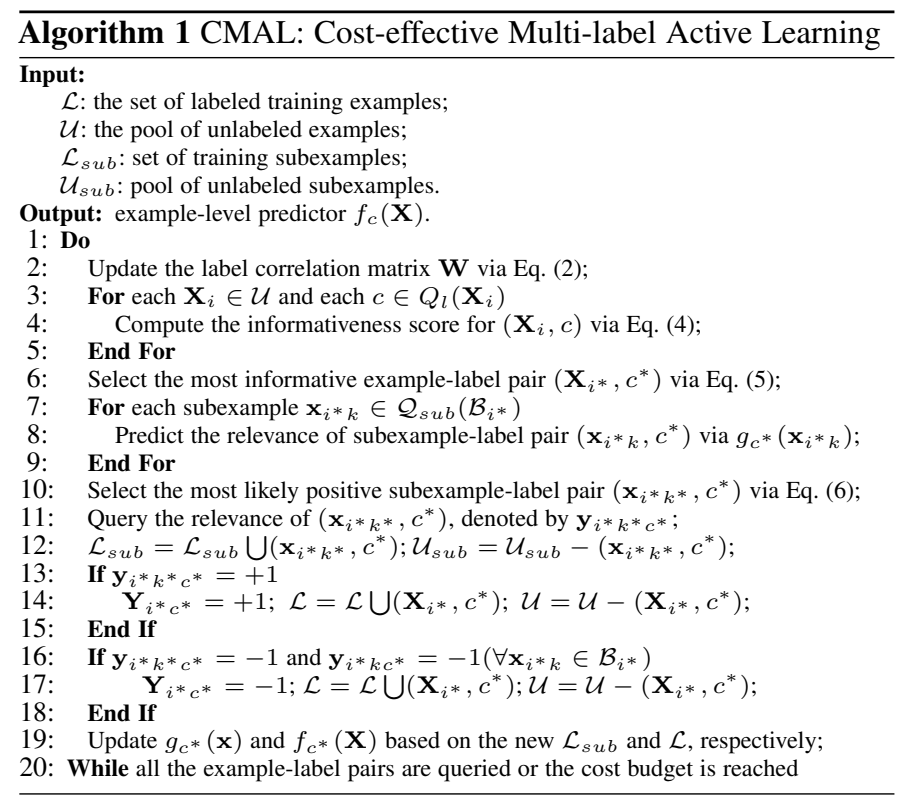

rCMAL integrates representative with informativeness to design a more effective example-label pair selection strategy, instead of only the informativeness used by CMAL.

To measure the representative of examples, we first apply clustering on all the labeled $(\mathcal{L})$ and unlabeled examples $(\mathcal{U})$ to explore their intrinsic structure. We adopt $k$-means clustering here for its simplicity and efficiency. Suppose $\mathcal{C}=\left\{\mathcal{C}_{1}, \mathcal{C}_{2}, \ldots, \mathcal{C}_{K}\right\}$ represents the final clustering results, where $K$ is the number of clusters and $\mathcal{C}_{k}$ represents the set of examples in the $k$-th cluster. The cluster center of the $k$-th cluster is formalized as follows:

$$
\mathbf{u}_{k}=\frac{\sum_{\mathbf{X}_{i} \in \mathcal{C}_{k}} \mathbf{X}_{i}}{\left|\mathcal{C}_{k}\right|}
$$

where $\left|\mathcal{C}_{k}\right|$ represents the number of examples in $\mathcal{C}_{k}$. After that, an intuitive idea to measure the representative of each example is to use the accumulated representative of the example with respect to each cluster. In addition, considering the informativeness involved in clusters, clusters with more information should be more beneficial to improve the classification model. From this perspective, following the previous example-label pairs based query setting, we develop an informativeness-weighted accumulation method to measure the representative score of example-label pairs as follows:

$$
R\left(\mathbf{X}_{i}, c\right)=\sum_{k=1}^{K} \varphi\left(\mathcal{C}_{k}, c\right) \rho\left(\mathbf{X}_{i}, \mathcal{C}_{k}\right)
$$

where

$$
\begin{gathered}
\varphi\left(\mathcal{C}_{k}, c\right)=\frac{1}{\left|f_{c}\left(\mathbf{u}_{k}\right)\right|} \\
\rho\left(\mathbf{X}_{i}, \mathcal{C}_{k}\right)=\exp \left(-\left\|\mathbf{X}_{i}-\mathbf{u}_{k}\right\|_{2}^{2}\right)
\end{gathered}
$$

$\varphi\left(\mathcal{C}_{k}, c\right)$ represents the informativeness score of the $k$-th cluster with respect to the $c$-th label, and $\rho\left(\mathbf{X}_{i}, \mathcal{C}_{k}\right)$ denotes the representative score of example $\mathbf{X}_{i}$ with respect to the $k$-th cluster. Given the cluster center representation $\mathbf{u}_{k}$, Eq. (9) calculates the informativeness of the cluster-label pair $\left(\mathcal{C}_{k}, c\right)$ based on its uncertainty, using the same way as measuring the uncertainty of example-label pairs in Eq. 11. For the representative of example-cluster pair $\left(\mathbf{X}_{i}, \mathcal{C}_{k}\right)$, we assume that the closer the example $\mathbf{X}_{i}$ to the cluster center $\mathbf{u}_{k}$, the more representative $\mathbf{X}_{i}$ to $\mathcal{C}_{k}$ is; say $\mathbf{X}_{i}$ can better represent 
the overall input patterns of examples in $\mathcal{C}_{k}$. Following this idea, we define Eq. (10) to compute the representative of examplecluster pairs. In particular, Eq. (10) corresponds to a Laplacian Kernel function $\rho\left(\mathbf{X}_{i}, \mathcal{C}_{k}\right)=\exp \left(-\frac{\left\|\mathbf{X}_{i}-\mathbf{u}_{k}\right\|}{\sigma}\right)$ with Gaussian kernel width parameter $\sigma=1$. Other appropriate methods to measure the representative of example-cluster pairs can be studied in future work. Note that, although we use $R\left(\mathbf{X}_{i}, c\right)$ to quantify the representative score of the example-label pair $\left(\mathbf{X}_{i}, c\right), R\left(\mathbf{X}_{i}, c\right)$ also implies the informativeness of $\left(\mathbf{X}_{i}, c\right)$ in cluster level, thanks to the employment of $\varphi\left(\mathcal{C}_{k}, c\right)$.

Given the representative score $R\left(\mathbf{X}_{i}, c\right)$ and informativeness score $\tilde{U}\left(\mathbf{X}_{i}, c\right)$ of example-label pairs, a simple way to integrate representative with informativeness is to combine these two scores linearly using weights. However, the weight parameter seems very sensitive to different datasets (based on our preliminary study). Given that, we adopt an ad-hoc method here. Particularly, rCMAL first selects top $\theta$ example-label pairs with the highest $\tilde{U}\left(\mathbf{X}_{i}, c\right)$ as the candidates $\mathcal{X}_{\theta}$, and then selects the example-label pair with the highest $R\left(\mathbf{X}_{i}, c\right)$ from $\mathcal{X}_{\theta}$ for query. This process is formalized as follows:

$$
\left(\mathbf{X}_{i^{*}}, c^{*}\right)=\arg \max _{\mathbf{X}_{i} \in \mathcal{X}_{\theta}, c \in \mathcal{Q}_{l}\left(\mathbf{X}_{i}\right)} R\left(\mathbf{X}_{i}, c\right)
$$

The pseudo-code of rCMAL is summarized in Algorithm 2

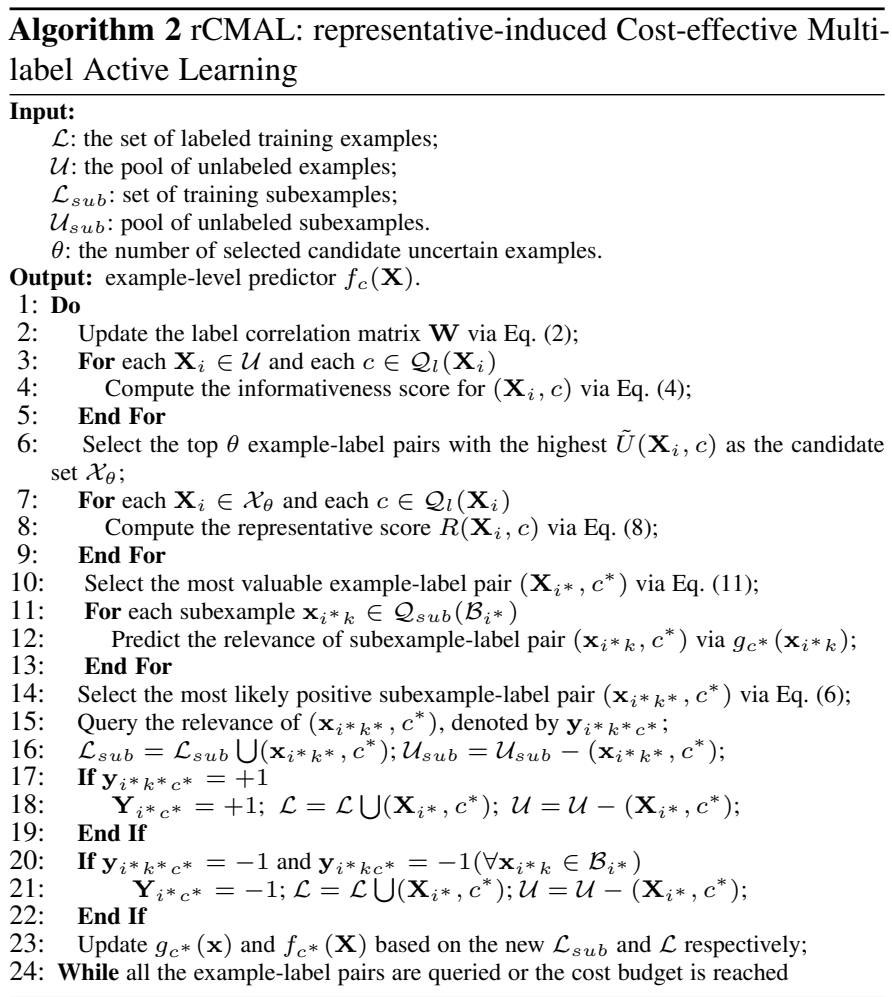

\section{EXPERIMENTS}

\subsection{Experimental Setup}

Datasets: To validate the effectiveness of our proposed CMAL and $\mathrm{rCMAL}$, the subexample-level labels are required to simulate the oracle (annotator). Under this restriction, we collected four eligible and widely-used datasets for our experiments [23], [54], whose statistical information are listed in Table 1 In addition, we also simulate four additional datasets (ImageK2, ImageK5, SceneK2 and SceneK5) by taking the original examples as subexamples, and performing $k$-means (with $k=(l+u) / 2$ and $k=(l+u) / 5)$ on the original multi-label datasets (Image [55] and Scene [56]) to generate new examples. Birds is a multi-label dataset segmented for multi-instance multi-label learning [54]. Each bag of this dataset corresponds to a 10 second recording of bird songs, and the recording is temporally segmented to create instances. There are 10,232 instances represented by 38dimensional feature vectors and 13 types of birds, and each type is considered as a particular label. MSRC-v2 [57] is a subset of the Microsoft Research Cambridge (MSRC) image datase 1 It contains 591 images (bags) and 23 classes. Based on the groundtruth segmentation, histograms of gradients and colors are extracted to form instances of each image. Letter_carroll and Letter_frost [54] are two letter datasets constructed based on Letter Recognition dataset of UCI (University of California Irvine) Machine Learning Repository ${ }^{2}$, where a bag is created for each word, and labels correspond to the letters. Since the feature vectors of examples are unavailable for the four datasets, we followed the widely-adopted solution in [14] to generate them. Particularly, a bag of instance feature vectors are converted into a bag feature vector by calculating $\mathbf{X}_{i}=\left[\operatorname{sim}\left(\mathcal{B}_{i}, \mathbf{c}_{1}\right), \operatorname{sim}\left(\mathcal{B}_{i}, \mathbf{c}_{2}\right), \cdots, \operatorname{sim}\left(\mathcal{B}_{i}, \mathbf{c}_{d}\right)\right]$, where $\mathbf{c}_{1}, \mathbf{c}_{2}, \cdots, \mathbf{c}_{d}$ are $d$ cluster centers obtained by $k$-means $(k=d)$ on all instances and $\operatorname{sim}\left(\mathcal{B}_{i}, \mathbf{c}\right)=\min _{\mathbf{x} \in \mathcal{B}_{i}} \exp \left(-\frac{\|\mathbf{x}-\mathbf{c}\|_{2}^{2}}{\delta}\right) . d$ is fixed to $50 \%$ of the bags and $\delta$ is set to the average distance between instances of the cluster. Other solutions can also be adopted to generate the feature vectors of bags [58], [59]. In this paper, bags and instances are called as examples and subexamples, respectively. Compared Methods: To comparatively study the performance, we take five state-of-the-art MAL methods as comparing methods:

(i) CVIRS (Category Vector Inconsistency and Ranking of Scores) [6] selects the most valuable example by incorporating two uncertainty measures: one is based on a rank aggregation of difference margins of classifier predictions, and the another is based on the inconsistency of predicted label sets.

(ii) QUIRE (QUerying Informative and Representative Examples) [10] selects the most valuable example-label pair with both high informativeness and representative.

(iii) AGIN (AGgregated INformativeness) [21] measures the uncertainty of each bag by aggregating the prediction uncertainty of its instances, and then queries the bag with the highest uncertainty.

(iv) MidSelect [22] first converts multi-instance multi-label examples into single-instance representations, and then selects the most uncertain examples for query.

(v) MIML-AL (Multi-Instance Multi-Label Active Learning) [23] first selects the most valuable example-label pair using diversity and uncertainty, and additionally requires the annotator to identify the key subexample of this example-label pair once the pair is deemed as positive.

These approaches employ different query strategies. At each iteration, CVRIS, AGIN and MidSelect query all labels for one selected example; QUIRE and MIML-AL query the relevance of one selected example-label pair. These methods query the examples or example-label pairs based on all subexamples of the selected example. CMAL and rCMAL query the relevance of an examplelabel pair by querying its selected subexample-label pairs. For a fair comparison, $q$ one-vs-rest SVMs (implemented with LIBSVM [60]) are used as the basic classification model to evaluate all the

1. http://research.microsoft.com/vision/cambridge/recognition/

2. http://archive.ics.uci.edu/ml/datasets.php/ 
TABLE 1

Summary of benchmark datasets. AvgLabels is the average number of labels per bag, Min/Avg/Max are the minimum/average/maximum numbers of subsexamples of all examples of the dataset.

\begin{tabular}{l|crrrrrr}
\hline & Dataset & Examples & Subexamples & Features & Labels & AvgLabels & Min/Avg/Max \\
\hline \multirow{4}{*}{ Widely used } & Birds & 548 & 10232 & 38 & 13 & 2.1 & $2 / 18.7 / 43$ \\
& MSRC-v2 & 591 & 1758 & 48 & 23 & 2.5 & $1 / 3.0 / 17$ \\
& Letter_carroll & 166 & 717 & 16 & 26 & 3.9 & $1 / 4.3 / 12$ \\
& Letter_frost & 144 & 565 & 16 & 26 & 3.6 & $1 / 3.9 / 11$ \\
\hline \multirow{5}{*}{ Simulated } & ImageK2 & 1000 & 2000 & 294 & 5 & 1.6 & $1 / 2.0 / 18$ \\
& ImageK5 & 400 & 2000 & 294 & 5 & 2.2 & $1 / 5.0 / 42$ \\
& SceneK2 & 1204 & 2407 & 294 & 5 & 1.1 & $1 / 2.0 / 27$ \\
& SceneK5 & 481 & 2407 & 294 & 5 & 1.4 & $1 / 5.0 / 43$ \\
\hline
\end{tabular}

approaches. The parameters $C$ (penalty of the error term) and $\gamma$ (RBF kernel parameter) of SVMs used in our experiments are tuned via 5 -fold cross-validation on the initial set. Particularly, for rCMAL, we simply set $K=\lceil u / q\rceil$ for $k$-means clustering, and $\theta=2 * q$ for selecting the candidate example-label pairs. For each experiment, we randomly divide the dataset into three parts: the test set with $50 \%$ of the examples, the initial labeled set with $5 \%$ of the examples, and the unlabeled pool with the rest $(50 \%)$ of the examples. The subexamples of all the examples are also accordingly divided into three parts. We repeat the random data partition 10 times, and report the average results. After each query, we update the predictor on the augmented labeled data and evaluate its performance on the test set. When all the examples in the unlabeled pool have been queried, the query process terminates. Evaluation Metrics: We adopt two representative and widelyused multi-label learning evaluation metrics: RankingLoss and AveragePrecision [17]. RankingLoss evaluates the average fraction of label pairs that are not correctly ranked, and AveragePrecision evaluates the average fraction of labels ranked above a particular label of the example. The smaller the values of RankingLoss, the better the performance is, while the larger the values of AveragePrecision, the better the performance is. We report 1 RankingLoss and AveragePrecision in the following experiments. As such, larger values imply a better performance.

\subsection{Comparison against State-of-the-art Methods}

To enable quantitative comparison, here we follow the assumption that querying all $q$ labels for one example is equivalent to querying $q$ example-label pairs. This assumption has been widely used in example-label pair based multi-label active learning $[\overline{8}]-[10]$. Similarly, we assume that querying all subexamples of one example is equivalent to querying the whole example. Based on this setup, we report the 1-RankingLoss and AveragePrecision of all the methods in Figures $2 \sqrt{3}$ as the number of queried subexamplelabel pairs increase.

Based on the results in these two figures, we have the following observations:

(i) subexample-label vs. example-label pair. Both CMAL and rCMAL manifest a better performance than QUIRE and MIML$\mathrm{AL}$, which select example-label pair for querying. That is because CMAL and rCMAL surrogate the query of an example-label pair with its subexample-label pairs, each of which has a lower cost than the whole example-label pair. QUIRE has to query the relevance between all subexamples and a label. As a result, it also asks for more cost than CMAL and rCMAL for achieving the same performance. This fact confirms the effectiveness of our two-stage active learning and the rationality of subexample-label pair query. (ii) example vs. example-label pair. AGIN, CVRIS and MidSelect select the most valuable examples and query the relevance between example with respect to all labels. To achieve a similar performance, they often take more costs. Although MIML-AL also queries the relevance of example-label pair, it has the lowest 1-RankingLoss and AveragePrecision values, that is because it doesn't use the example-level features, but all the other methods do.

(iii) informative vs. informative +representative. rCMAL achieves a better (or comparable) performance than CMAL in most cases (on Birds, MSCV2, Letter_frost, Letter_carroll, ImageK2 and ImageK5 datasets), which proves the contribution of representative for MAL, and the effectiveness of rCMAL. However rCMAL loses to CMAL on SceneK2 and SceneK5 datasets. The reason may be that the way of capturing the representative of example-label pairs or the way of integrating informativeness and representative are not suitable for these two datasets.

To further study the potential of CMAL and rCMAL, we count the number of performed queries of all methods when the test performance reaches $95 \%$ of the best performance (that is, the performance at the end of the performance curves in Figure 2 33, and report the results in Table 2. We can find that: (i) CMAL and rCMAL significantly reduces the number of queries in most cases; (ii) The reduced number of queries is more significant on Birds, ImageK5 and SceneK5. The reason is that the average number and the maximum number of subexamples per example of these three datasets are smaller than that of Birds, as seen in Table 1 From these observations, we can conclude that CMAL and rCMAL can achieve a superior performance ( $95 \%$ of the best performance) by querying a far fewer number of subexample-label pairs than the comparing methods. For example, to reach $95 \%$ of the best AveragePrecision on the Birds dataset, rCMAL needs only 34\%, $16 \%, 29 \%, 31 \%$ and $17 \%$ of the queries required by CVIRS, AGIN, QUIRE, MidSelect and MIML-AL, respectively.

From these results, we can conclude that performing multi-label active learning by querying subexamples of the selected examplelabel pair can achieve a superior performance with less cost than other methods that query examples or example-label pairs.

\subsection{Impact of Different Query Costs}

In the previous experiments, we reported the performance curves based on the assumption that the total number of queries for each example $\mathbf{X}_{i}$ is $n_{i} \times q$, where $n_{i}$ is the number of subexamples of $\mathbf{X}_{i}$ and $q$ is the number of distinct labels. However, this assumption may be too optimistic. There is a typical problem where context and perception play an important role in annotating the example. First, for a particular example-label pair, the cost of annotating all its subexample-label pairs may be not the same. More exactly, the more subexample-label pairs of the example-label pair are queried, the more the annotator know about this pair, and thus the lower the cost for querying the rest of un-queried subexample-label pairs is. Second, the cost of iteratively querying all subexample-label pairs 


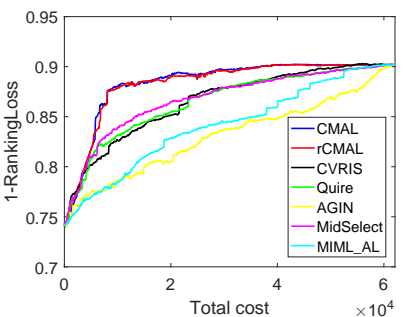

(a) Birds

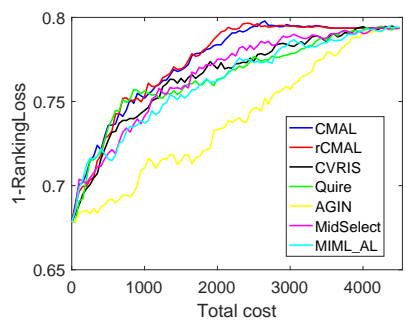

(e) ImageK2

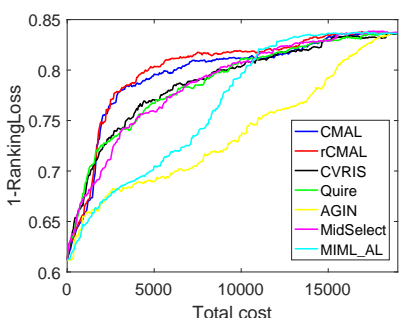

(b) MSRC-v2

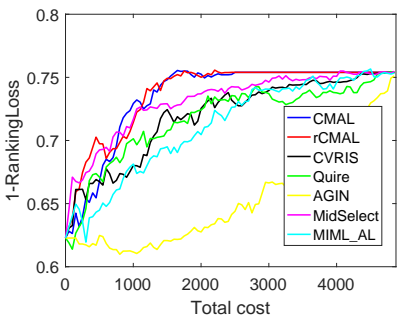

(f) ImageK5

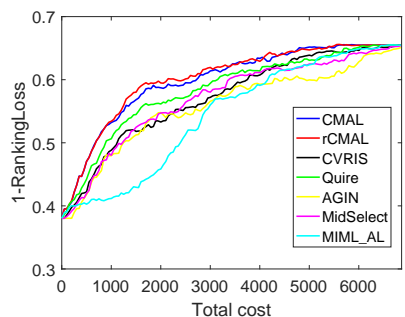

(c) Lettet_carroll

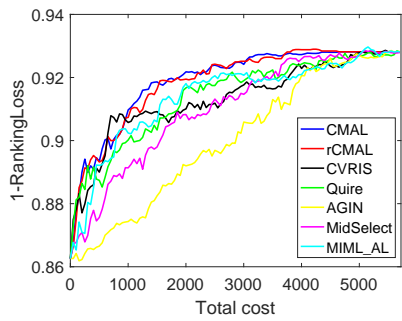

(g) SceneK2

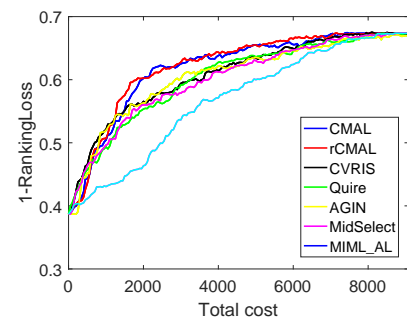

(d) Lettet_frost

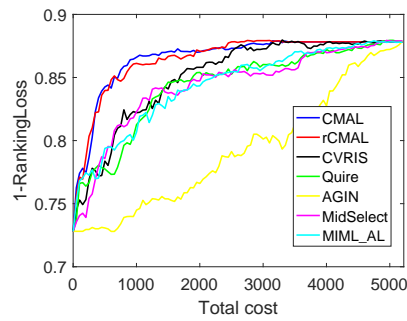

(h) SceneK5

Fig. 2. 1-RankingLoss vs. total query cost (i.e., the total number of queries of subexample-label pairs).

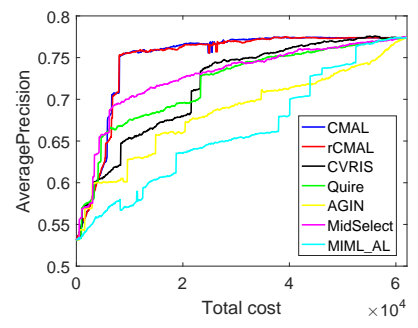

(a) Birds

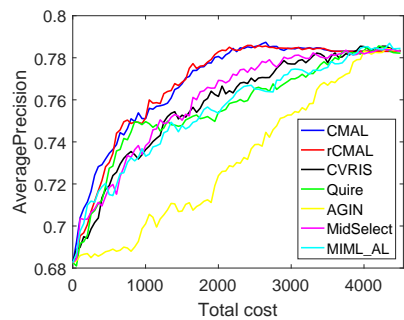

(e) ImageK2

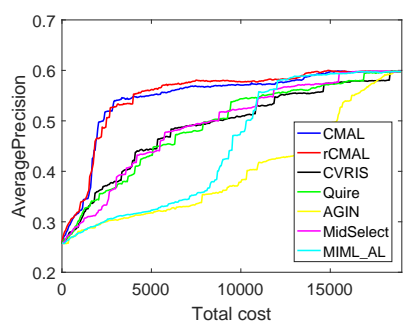

(b) MSRC-v2

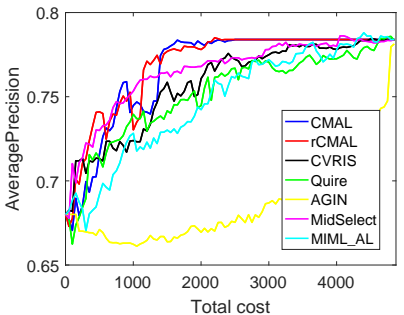

(f) ImageK5

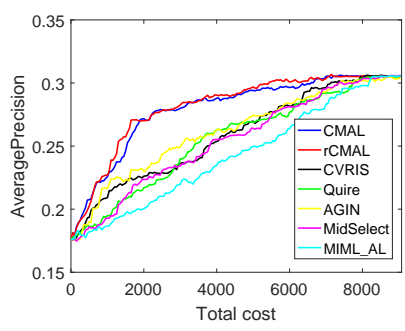

(c) Lettet_carroll

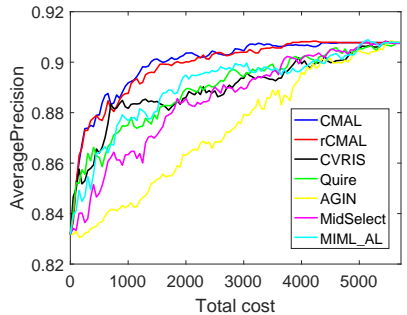

(g) SceneK2

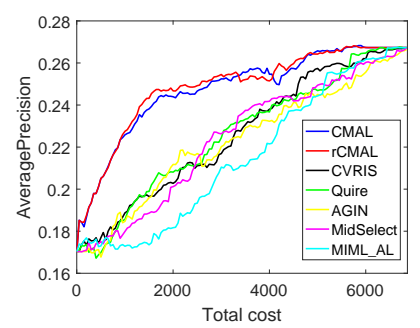

(d) Lettet_frost

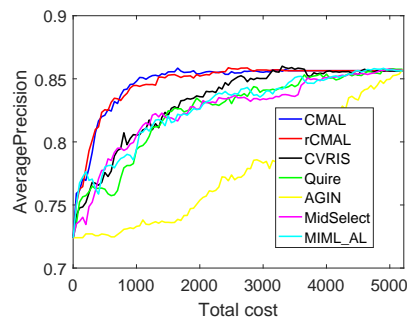

(h) SceneK5

Fig. 3. AveragePrecision vs. total query cost (i.e., the total number of queries of subexample-label pairs).

of one selected example-label pair may be higher than scrutinizing the whole example for the label. Unfortunately, there is no off-theshelf datasets with individual subexample-level and example-level costs. In the following, we simply simulate the above two actual scenarios, and then conduct additional experiments under these scenarios to further investigate the effectiveness of CMAL and rCMAL with varying costs of subexample-label pairs.

For the first scenario where the cost of annotating all subexample-label pairs of one selected example-label pair may be not the same, we formulate a manner to measure the query cost of subexample-label pairs. Firstly, we define an auxiliary function as follows:

$$
\psi(\lambda)=\alpha^{-\lambda}
$$

where $\alpha>1$ is the user-defined decay parameter that gradually assigns a smaller query cost as more subexample-label pairs are queried. For illustration, we plot the curve of this function $\psi(\lambda)=$ $\alpha^{-\lambda}$ with $\alpha \in\{1.01,1.03,1.05\}$ in Figure 4 After that, we define the cost of querying the $b$-th (the first $(b-1)$ subexample-label pairs already queried) subexample-label pair $\left(\mathbf{x}_{i k}, c\right)$ of $\left(\mathbf{X}_{i}, c\right)$ as follows:

$$
\Omega\left(\mathbf{x}_{i k}, c, b\right)=\frac{\int_{b-1}^{b} \psi(\lambda) d \lambda}{\int_{0}^{\left|\mathcal{B}_{i}\right|} \psi(\lambda) d \lambda} * \Omega\left(\mathbf{X}_{i}, c\right)
$$

where $\left|\mathcal{B}_{i}\right|$ is the total number of subexamples of $\mathbf{X}_{i}$, and $\Omega\left(\mathbf{X}_{i}, c\right)$ is the query cost of example-label pair $\left(\mathbf{X}_{i}, c\right)$. To enable quantitative comparison, we follow a canonical assumption that $\Omega\left(\mathbf{X}_{i}, c\right)=1$ for any example-label pair $[8]-[10]$ for the following simulated experiments. $\int_{b-1}^{b} \psi(\lambda) d \lambda$ and $\int_{0}^{\left|\mathcal{B}_{i}\right|} \psi(\lambda) d \lambda$ denote the definite integral of function $\psi(\lambda)$ in the range of $[b-1, b]$ and $\left[0,\left|\mathcal{B}_{i}\right|\right]$, respectively. From Figure 4 , we observe that, a larger $b$ leads to a smaller $\int_{b-1}^{b} \psi(\lambda) d \lambda$ given $\alpha>1$, thus a smaller cost 
TABLE 2

Comparison of CMAL/rCMAL against the competitive methods on the total number of queries for subexample-label pair when the test performance reaches $95 \%$ of the best performance (i.e., the performance at the end of the curves in Figures 23. The smallest number of queries for subexample-label pair are in boldface.

\begin{tabular}{|c|c|c|c|c|c|c|c|c|c|c|}
\hline & & CVRIS & AGIN & Quire & MidSelect & MIML-AL & rCMAL(RR) & rCMAL(RM) & CMAL & rCMAL \\
\hline \multirow{2}{*}{ Birds } & RankingLoss & 21568 & 46138 & 21415 & 16433 & 37994 & 18753 & 14840 & 6651 & 6761 \\
\hline & AveragePrecision & 23674 & 50012 & 27766 & 26092 & 46130 & 23332 & 22438 & 8083 & 8012 \\
\hline \multirow{2}{*}{ MSRC-v2 } & RankingLoss & 8143 & 15273 & 8354 & 7959 & 9986 & 8059 & 6447 & 4966 & 4088 \\
\hline & AveragePrecision & 14629 & 17113 & 14323 & 12605 & 12024 & 12612 & 12454 & 7104 & 5593 \\
\hline \multirow{2}{*}{ Letter_carroll } & RankingLoss & 5201 & 5669 & 5242 & 5565 & 6375 & 5022 & 4703 & 3652 & 3707 \\
\hline & AveragePrecision & 6397 & 6709 & 6886 & 6839 & 7295 & 6504 & 6218 & 4548 & 3747 \\
\hline \multirow{2}{*}{ Letter_frost } & RankingLoss & 4317 & 5799 & 4172 & 4889 & 4865 & 4088 & 3908 & 3412 & 3298 \\
\hline & AveragePrecision & 4759 & 5799 & 5474 & 5487 & 5169 & 4675 & 4409 & 3326 & 2938 \\
\hline \multirow{2}{*}{ ImageK2 } & RankingLoss & 1221 & 2796 & 825 & 1191 & 1500 & 1064 & 928 & 899 & 676 \\
\hline & AveragePrecision & 1196 & 2646 & 779 & 1071 & 1250 & 1040 & 927 & 651 & 601 \\
\hline \multirow{2}{*}{ ImageK5 } & RankingLoss & 1401 & 4256 & 1705 & 996 & 2168 & 932 & 858 & 851 & 977 \\
\hline & AveragePrecision & 1331 & 4711 & 1583 & 686 & 1927 & 697 & 682 & 768 & 484 \\
\hline \multirow{2}{*}{ SceneK2 } & RankingLoss & 146 & 1486 & 103 & 591 & 321 & 205 & 205 & 113 & 123 \\
\hline & AveragePrecision & 446 & 1946 & 295 & 671 & 447 & 213 & 209 & 154 & 125 \\
\hline \multirow{2}{*}{ SceneK5 } & RankingLoss & 1396 & 4016 & 1229 & 1136 & 1492 & 913 & 862 & 405 & 450 \\
\hline & AveragePrecision & 1191 & 4016 & 1371 & 1131 & 1256 & 743 & 741 & 385 & 369 \\
\hline
\end{tabular}

of querying the $b$-th subexample-label pair. In other words, the query cost for the remaining un-queried subexample-label pairs of $\left(\mathbf{X}_{i}, c\right)$ decreases, as more subexample-label pairs have been queried. In addition, a larger $\alpha$ leads to a faster slope of query cost. We simply set $\alpha=1.03$ for the first scenario, and report the results in Figure 5 and Figure S1 of the supplementary file.

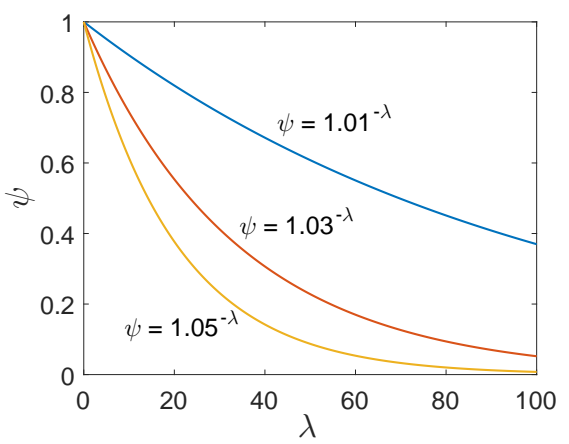

Fig. 4. The curves of auxiliary function $\psi(\lambda)=\alpha^{-\lambda}$ with $\alpha \in$ $\{1.01,1.03,1.05\}$.

For the second scenario where the cost of iteratively querying all subexample-label pairs of a selected example-label pair is higher than that of directly querying the example-label pair, we slightly modify $\Omega\left(\mathbf{x}_{i k}, c, b\right)$ as follows:

$$
\Omega\left(\mathbf{x}_{i k}, c, b\right)=\beta \frac{\int_{b-1}^{b} \phi(\lambda) d \lambda}{\int_{0}^{\left|\mathcal{B}_{i}\right|} \phi(\lambda) d \lambda} * \Omega\left(\mathbf{X}_{i}, c\right)
$$

where $\beta$ is a parameter that controls the scale between the cost of iteratively querying all subexample-label pairs of one selected example-label pair and the cost of directly querying the examplelabel pair. For example, $\beta=1.1$ means the cost of iteratively querying all subexample-label pairs is 1.1 times of directly querying the example-label pair. For simulated analysis, we conduct experiments with $\beta \in\{1.1,1.2,1.3\}$. To reduce redundancy, we only report the results with $\beta=1.1$ in Figure 6 and Figure S2 of the supplementary file. Note that, the query cost of each examplelabel pair is fixed to 1 in Figure 5 and Figure S1-S2, while the previous experiments fixed the query cost of each subexample-label pair to 1 .

From the results in Figure 5,6 and Figure S1-S2, we have the following observations: (i) CMAL and rCMAL have a better performance than other compared methods under the same cost, even with different costs for different subexample-label pairs, or with a larger cost for querying subexample-label pairs than the example-label pair. Compared the results with those in Figure 2 23, the performance gap between CMAL (rCMAL) and the other methods reduces. A larger $\alpha$ reduces the performance gap between CMAL (rCMAL) and other compared methods, since a larger $\alpha$ implies a larger cost of querying the initially selected subexample-label pairs, which increases the cost of querying the target example-label pair. Similarly, a large $\beta$ also increases the cost of querying subexample-label pairs, and thus reduces the performance margin between CMAL (rCMAL) and other compared methods. In practice, we also observe that CMAL (rCMAL) still significantly outperforms the other compared methods in most cases with $\beta=1.2$ or $\beta=1.3$, which further demonstrate the effectiveness of CMAL/rCMAL. (ii) CMAL and rCMAL have an obviously smaller cost than other compared methods at the end of the curve on Birds and Letter_carroll datasets (all example-label pairs in the pool are queried). The reason is that CMAL and rCMAL query the example-label pair by querying its subexample-label pairs as few as possible, and thus save the cost. This observation attests that CMAL and rCMAL can achieve a higher classification performance than other related methods at a lower cost. (iii) In Figure 6 and Figure S2, all compared methods have similar query costs at the end of the curve. The reason is that we assume the query cost of iteratively querying all subexample-label pairs of one example-label pair is 1.1 times of directly querying the examplelabel pair, which reduces the cost gap between CMAL (rCMAL) and other compared methods.

Overall, we have the similar conclusions as those from Figure 2 . 3 which again confirms the effectiveness of the proposed two-stage active learning.

\subsection{Ablation Study}

\subsubsection{Effectiveness of Individual Stage}

Both CMAL and rCMAL refer to two stages for querying the labels of examples: the first stage for selecting the most valuable example-label pairs, and the second stage for querying the most likely positive subexample-label pair of the selected example-label pair. Note that, the second stage of CMAL and RCMAL is the same. To validate the effectiveness of each individual stage in rCMAL, we introduce two variants: (i) $\operatorname{rCMAL}(\mathrm{RM})$ first randomly selects the example-label pair, and then chooses the most likely 


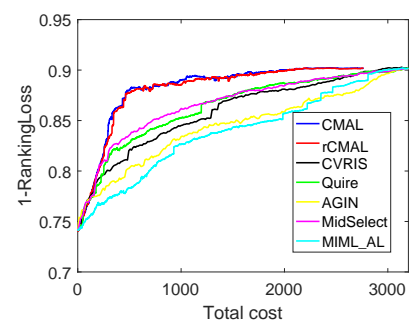

(a) Birds

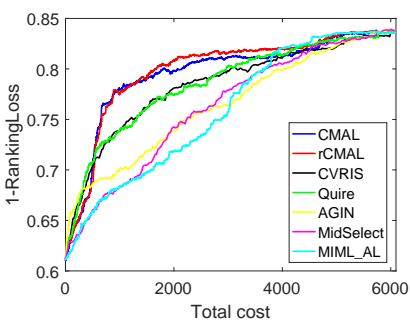

(b) MSRC-v2

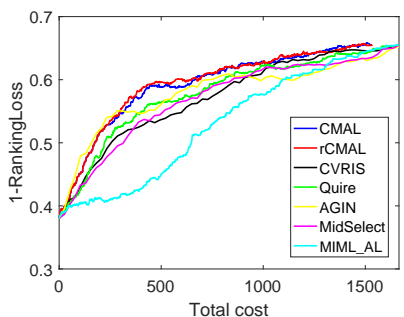

(c) Lettet_carroll

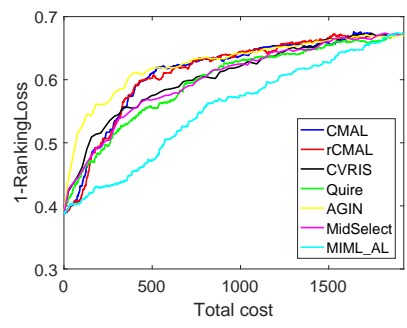

(d) Lettet_frost

Fig. 5. 1-RankingLoss vs. total query cost, the query cost for subexample-label pairs of the same example-label pair is gradually reduced.

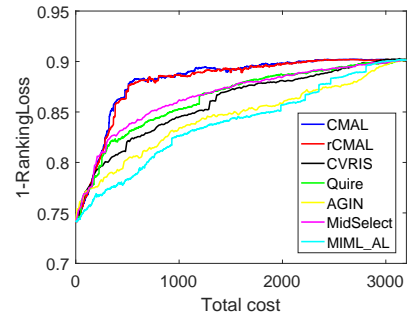

(a) Birds

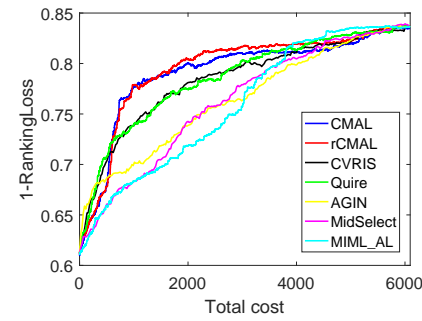

(b) MSRC-v2

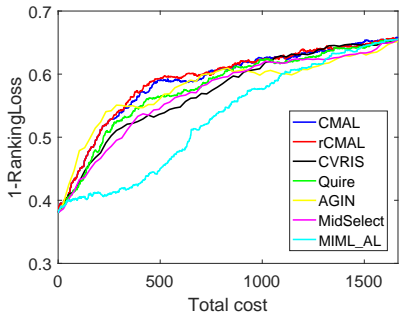

(c) Lettet_carroll

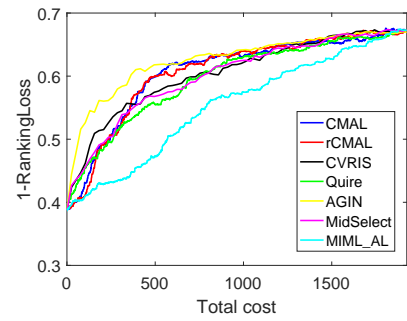

(d) Lettet_frost

Fig. 6. 1-RankingLoss vs. total query cost, the total query cost of all subexample-label pairs is larger than the example-label pair.

positive subexample-label pair of the selected example-label pair for the query; (ii) rCMAL(RR) first randomly selects the examplelabel pair and then randomly chooses subexample-label pairs of the selected example-label pair for the query. Following the experimental setup in Section 4.2. we compare our rCMAL against these two variants, and report the results on four real-world datasets in Figure 7 and Figure S3 of the supplementary file.

Based on the results in these two figures, we can make the following conclusions:

(i) Querying the informative and representative example-label pairs contributes to a significantly improved performance and reduced the query cost. rCMAL(RM) does not select the informative and representative example-label pairs for the second stage, while rCMAL does. As a result, rCMAL significantly outperforms rCMAL(RM) in terms of 1-RankingLoss and AveragePrecision. In addition, $\mathrm{rCMAL}(\mathrm{RM})$ needs much more queries to reach a comparable performance with rCMAL.

(ii) Selecting the most likely positive subexample-label pair for query is rational and effective. This conclusion is supported by the observation that $\mathrm{rCMAL}(\mathrm{RM})$ outperforms $\mathrm{rCMAL}(\mathrm{RR})$ in most cases. $\mathrm{rCMAL}(\mathrm{RM})$ chooses the most plausible positive subexample-label pair for query, while $\mathrm{rCMAL}(\mathrm{RR})$ randomly chooses a subexample-label pair for query. This ablation study further confirms the effectiveness and rationality of our two-stage rCMAL.

\subsubsection{Contribution of Label Correlation and Label Space Sparsity}

The characteristics of label correlation and label space sparsity should be considered in effective multi-label active learning. To study the contribution of label correlation and label space sparsity to rCAML, three variants of rCMAL are introduced: (i) rCMAL(nC) does not consider the label correlation; (ii) $\operatorname{rCMAL}(\mathrm{nS})$ does not consider the label space sparsity; (iii) $\operatorname{rCMAL(nCS)}$ neither considers the label correlation nor the label space sparsity.
Figure 8 and Figure S5 of the supplementary file give the performance curves of rCMAL and of three variants on four realworld datasets, respectively. We have the following observations: (i) $\operatorname{rCMAL}(\mathrm{nS})$ frequently has a lower performance in terms of 1RankingLoss and AveragePrecision than rCMAL. This performance margin confirms the contribution of label space sparsity for selecting valuable example-label pairs.

(ii) Compared with rCMAL(nC), rCMAL makes extra use of label correlation, and it often outperforms $\mathrm{rCMAL}(\mathrm{nC})$. This observation justifies the contribution of label correlation for selecting valuable example-label pairs. We also note that $\mathrm{rCMAL}(\mathrm{nC})$ and $\mathrm{rCMAL}$ hold a similar performance at the beginning, and their performance margin becomes obvious as more subexample-label pairs queried, especially on Birds and Letter_Carroll datasets. The reason may be that the initial label correlation cannot be well estimated with the scanty labeled training examples. As more examples queried, the estimated correlation becomes more reliable, and the contribution of label correlation becomes more prominent. As the further increase of queried examples, the gap between $\mathrm{rCMAL}(\mathrm{nC})$ and rCMAL gradually shrinks, since the relevance of most examplelabel pairs are queried, the benefit of label correlation for reducing the uncertainty of other un-queried labels gradually diminishes. (iii) rCMAL(nC) and rCMAL obtain the similar performance on MSRC-v2 and Letter_frost datasets. The cause may be that the used public datasets only have a small number of examples, and the average number of labels per example is also small. Thus, the learned label correlation may be inaccurate. This fact suggests the reliable label correlation and its proper usage can further boost the performance of multi-label active learning, which is an interesting future pursue.

(iv) $\mathrm{rCMAL}(\mathrm{nCS})$ often loses to $\mathrm{rCMAL}(\mathrm{nC})$ and $\mathrm{rCMAL}(\mathrm{nS})$, not to mention rCMAL. This observation not only confirms the effectiveness of separately mining label correlations and label space sparsity for MAL, but also proves the benefit of jointly leveraging label correlations and label space sparsity for effective MAL.

In summary, this ablation study suggests that the label corre- 


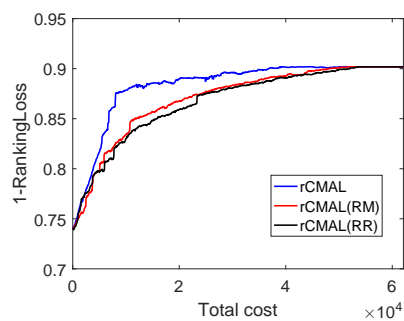

(a) Birds

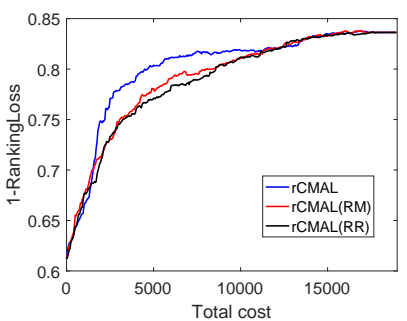

(b) MSRC-v2

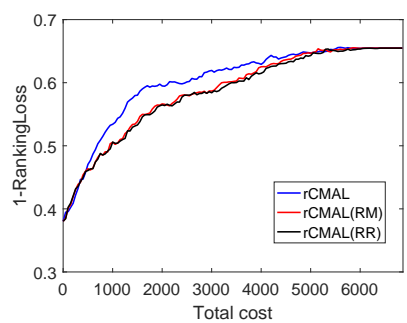

(c) Lettet_carroll

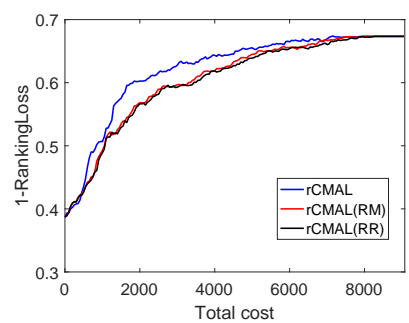

(d) Lettet_frost

Fig. 7. 1-RankingLoss vs. total query cost (i.e., the total number of queries of subexample-label pairs) of different variants.

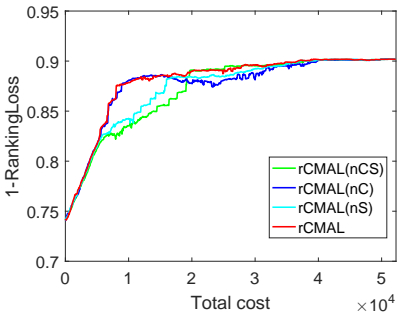

(a) Birds

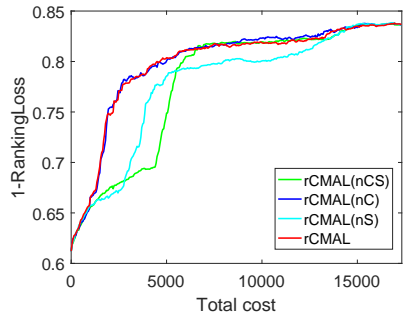

(b) MSRC-v2

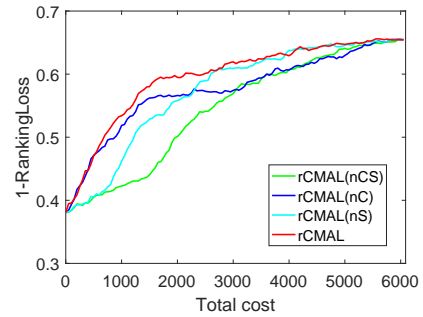

(c) Lettet_carroll

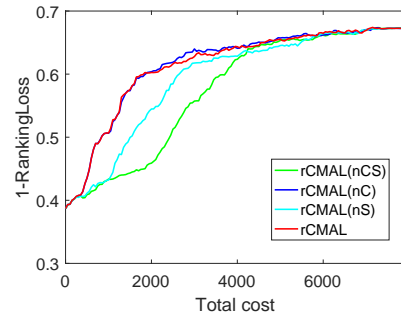

(d) Lettet_frost

Fig. 8. 1-RankingLoss vs. total number of queries of subexample-label pairs of different variants with/without label correlations and label sparsity.

lation and label space sparsity are important for MAL, and their proper usage can boost the performance of MAL.

\subsection{Results of Alternative Query Strategies}

CMAL and rCMAL are built on the canonical multi-instance learning principle that an example-label pair is negative if all its subexample-label pairs all get negative feedbacks. In this section, we investigate whether we can avoid the greedy query of all subexample-label pairs of negative example-label pairs. In fact, as more evidence of negative subexample-label pairs for a given example-label pair is accumulated, the larger the likelihod that this example-label will be a negative, since the most plausible positive subexample-label pairs are already queried with negative responses. In fact, we already have the subexample-level predictor $g_{c}(\mathbf{x})$, if $g_{c}(\mathbf{x})$ predicts all un-queried subexample-label pairs of the selected example as negative, then the likelihood that this example-label pair being negative increases. Therefore, we can avoid the excessive query of subexample-label pairs for an example-label pair with different options. For this purpose, we introduce five variants of rCMAL:

(i) $\operatorname{rCMAL}\left(\frac{n_{i}}{4}\right)$ annotates the selected example-label pair as negative if $\sum_{k=1}^{n_{i^{*}}}\left[\mathbf{y}_{i^{*}} k c^{*}=-1\right] \geq\left\lceil\frac{n_{i^{*}}}{4}\right\rceil$, namely the first quarter of queried subexamples of the example are all negative for the target label.

(ii) $\operatorname{rCMAL}\left(\frac{n_{i}}{2}\right)$ annotates the selected example-label pair as negative if $\sum_{k=1}^{n_{i^{*}}}\left[\mathbf{y}_{i^{*}} k c^{*}=-1\right] \geq\left\lceil\frac{n_{i^{*}}}{2}\right\rceil$, namely the first half of queried subexamples of the example are all negative for the target label.

(iii) $\operatorname{rCMAL}\left(\frac{n_{i}}{4}+C\right)$ annotates the selected example-label pair as negative if $\sum_{k=1}^{n_{i^{*}}}\left[\mathbf{y}_{i^{*}} k c^{*}=-1\right] \geq\left\lceil\frac{n_{i^{*}}}{4}\right\rceil$, or $\mathbf{y}_{i^{*}} k c^{*}=-1$ for all queried subexample-label pairs and $g_{c}(\mathbf{x})<0$ for all un-queried subexample-label pairs (consensus prediction).

(iv) $\operatorname{rCMAL}\left(\frac{n_{i}}{2}+C\right)$ annotates the selected example-label pair as negative if $\sum_{k=1}^{n_{i^{*}}}\left[\mathbf{y}_{i^{*}} k c^{*}=-1\right] \geq\left\lceil\frac{n_{i^{*}}}{2}\right\rceil$, or $\mathbf{y}_{i^{*}} k c^{*}=-1$ for all queried subexample-label pairs and $g_{c}(\mathbf{x})<0$ for all un-queried subexample-label pairs (consensus prediction).

(v) $\operatorname{rCMAL}\left(n_{i}+C\right)$ annotates the selected example-label pair as negative if $\mathbf{y}_{i^{*} k c^{*}}=-1$ for all queried subexample-label pairs, or $g_{c}(\mathbf{x})<0$ for all un-queried subexample-label pairs (consensus prediction).

Following the experimental setup in Section 4.2, we report the classification performance of rCMAL and of these variants as a function of the number of queried subexample-label pairs in Figure 9 and Figure S6 of the supplementary file. The end of a curve is due to all the example-label pairs in the pool are queried. The numbers of queried subexample-labels pairs (i.e., the costs) of these variants to inquire all example-label pairs in the same pool are different, since these variants differ in terminating the query of subexample-label pairs for the same example-label pair.

From these two figures, we can observe that (i) rCMAL costs more queries of subexample-label pairs than these variants, which do not excessively query all subexample-label pairs to annotate a negative example-label pair, and thus these variants greatly reduce the number of queries. However, they misjudge positive example-label pairs as negative, which will mislead the training of classifiers. (ii) Although rCMAL sometimes has a lower performance at the beginning, it has a greedily increased performance as the number of queries increase and outperforms these variants with a moderate cost in most cases. (iii) Incorporating the consensus prediction from subexample-level classifier reduce both the query cost and performance, since the consensus prediction brings in more misjudged negative example-label pairs. (iv) As $\theta=\{1,2,4\}\left(n_{i} / \theta\right)$ increases (decreases), no matter with or without the consensus of the subexample-level classifier, these variants misjudge more positive example-label pairs. This is explainable, the example-label pair can be more quickly deemed as negative as $\theta$ increases. As a result, more positive example-label pairs are wrongly deemed as negative, which result in a misled example-level classifier.

These alternative query strategies provide the user with options 


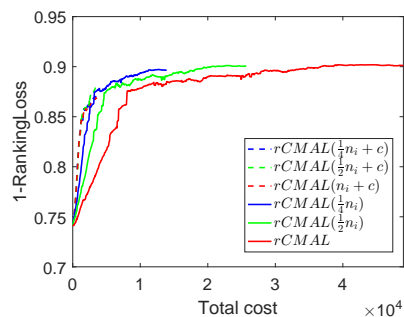

(a) Birds

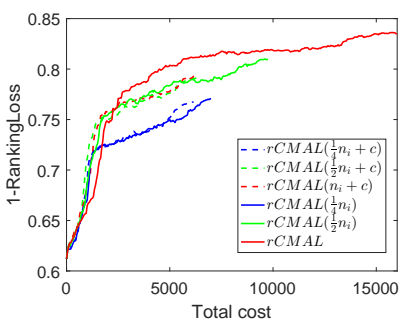

(b) MSRC-v2

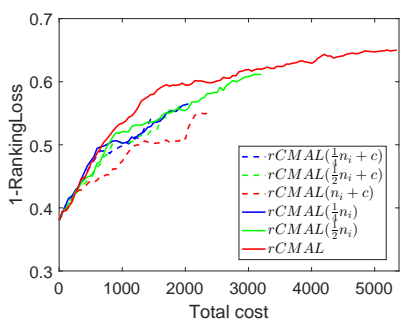

(c) Lettet_carroll

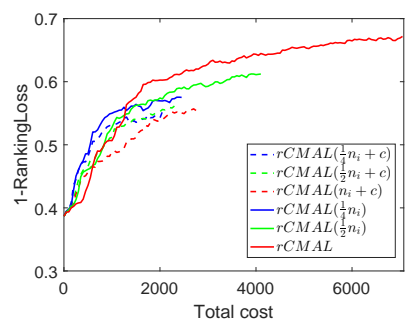

(d) Lettet_frost

Fig. 9. 1-RankingLoss vs. total number of queries of subexample-label pairs with different alternative query strategies.

to balance the query cost and performance improvement. If we have a small budget, the variant with a larger $\theta$ and consensus is suggested; otherwise, the variant with a smaller $\theta$ can be adopted.

In addition, we also study the time complexity and runtime of CMAL and rCMAL, and report them in the supplementary file. Overall, CMAL and rCMAL take more runtimes than other compared methods, they can reduce the number of precious human annotations and achieve a better (or comparable) performance at the expense of more computational times. How to further reduce the complexity while boost the performance is an interesting future work.

\section{Discussion And Conclusion}

We investigate how to reduce the query cost of multi-label active learning on examples (or bags) that can be segmented (or naturally represented) with multiple subexamples (instances), and assume the annotation cost for an example is larger than any of its subexamples. Based on this assumption, we propose two costeffective multi-label active learning algorithms called CMAL and rCMAL. Unlike existing example or example-label pair based active learning strategies, our algorithms surrogate the complex example-level query with subexample-level query with a reduced cost and complexity. Particularly, CMAL leverages uncertainty, label correlation and label space sparsity to jointly select the most informative example-label pairs for query, while rCMAL additionally uses the representative to select example-label pairs. Experimental results demonstrate that CMAL and rCMAL achieve a higher performance than other related methods at a lower query cost, and rCMAL achieves the best performance in most cases. We also prove the effectiveness of leveraging label correlation and label space sparsity for improving the selection of example-label pairs. However, we want to remark that inaccurate label correlations may not lead to an improvement. CMAL and rCMAL query the subexample-label pairs, instead of the example-label pairs. As a result, they take a larger computational cost for updating the subexample-level classifier, due to the number of subexamples is much larger than that of examples.

Other more appropriate techniques to leverage the informativeness and representative for selecting example-label pairs, and more ways for quantifying the individual query costs of subexample-label pairs and example-label pairs will be explored in the future work.

\section{References}

[1] B. Settles, "Active learning literature survey," Technical Report 1648, University of Wisconsin-Madison, 2009.

[2] M.-K. Xie and S.-J. Huang, "Learning class-conditional gans with active sampling," in $K D D, 2019$, pp. 998-1006.
[3] K. Brinker, "Incorporating diversity in active learning with support vector machines," in $I C M L, 2003$, pp. 59-66.

[4] C.-W. Hung and H.-T. Lin, "Multi-label active learning with auxiliary learner," in ACML, 2011, pp. 315-332.

[5] X. Li and Y. Guo, "Active learning with multi-label svm classification," in IJCAI, 2013, pp. 1479-1485.

[6] O. Reyes, C. Morell, and S. Ventura, "Effective active learning strategy for multi-label learning," Neurocomputing, vol. 273, pp. 494-508, 2018.

[7] O. Reyes and S. Ventura, "Evolutionary strategy to perform batch-mode active learning on multi-label data," TIST, vol. 9, no. 4, p. 46, 2018.

[8] G.-J. Qi, X.-S. Hua, Y. Rui, J. Tang, and H.-J. Zhang, "Two-dimensional multilabel active learning with an efficient online adaptation model for image classification," TPAMI, vol. 31, no. 10, pp. 1880-1897, 2009.

[9] S.-J. Huang and Z.-H. Zhou, "Active query driven by uncertainty and diversity for incremental multi-label learning," in ICDM, 2013, pp. 1079_ 1084.

[10] S.-J. Huang, R. Jin, and Z.-H. Zhou, "Active learning by querying informative and representative examples," TPAMI, vol. 36, no. 10, pp. 1936-1949, 2014.

[11] B. Zhang, Y. Wang, and F. Chen, "Multilabel image classification via high-order label correlation driven active learning," TIP, vol. 23, no. 3, pp. 1430-1441, 2014.

[12] S. J. Huang, J. L. Chen, X. Mu, Z. H. Zhou, S. J. Huang, J. L. Chen, $\mathrm{X}$. Mu, and $\mathrm{Z}$. H. Zhou, "Cost-effective active learning from diverse labelers," in IJCAI, 2017, pp. 1879-1885.

[13] Y.-F. Yan and S.-J. Huang, "Cost-effective active learning for hierarchical multi-label classification," in IJCAI, 2018, pp. 2962-2968.

[14] Y.-F. Li, J.-H. Hu, Y. Jiang, and Z.-H. Zhou, "Towards discovering what patterns trigger what labels," in AAAI, 2012, pp. 1012-1018.

[15] W. Liu and I. W. Tsang, "Making decision trees feasible in ultrahigh feature and label dimensions," JMLR, vol. 18, no. 1, pp. 2814-2849, 2017.

[16] H. T. Nguyen and A. Smeulders, "Active learning using pre-clustering," in $I C M L, 2004$, pp. 1-8.

[17] M. Zhang and Z. Zhou, "A review on multi-label learning algorithms," TKDE, vol. 26, no. 8, pp. 1819-1837, 2014.

[18] E. Gibaja and S. Ventura, "A tutorial on multilabel learning," ACM Comp. Surv., vol. 47, no. 3, p. 52, 2015.

[19] J. Tu, G. Yu, C. Domeniconi, J. Wang, G. Xiao, and M. Guo, "Multilabel crowd consensus via joint matrix factorization," KAIS, vol. 62, pp. $1341-1369,2020$.

[20] X. Chen, G. Yu, C. Domeniconi, J. Wang, Z. Li, and Z. Zhang, "Cost effective multi-label active learning via querying subexamples," in ICDM, 2018, pp. 905-910.

[21] M.-A. Carbonneau, E. Granger, and G. Gagnon, "Bag-level aggregation for multiple instance active learning in instance classification problems," TNNLS, vol. 30, no. 5, pp. 1441-1451, 2019.

[22] R. Retz and F. Schwenker, "Active multi-instance multi-label learning," in Analysis of Large and Complex Data, 2016, pp. 91-101.

[23] S.-J. Huang, N. Gao, and S. Chen, "Multi-instance multi-label active learning," in IJCAI, 2017, pp. 1879-1892.

[24] S. Tong and D. Koller, "Support vector machine active learning with applications to text classification," $J M L R$, vol. 2, no. 11, pp. 45-66, 2001.

[25] M.-F. Balcan, A. Broder, and T. Zhang, "Margin based active learning," in COLT, 2007, pp. 35-50.

[26] Y. Guo and D. Schuurmans, "Discriminative batch mode active learning," in NeurIPS, 2008, pp. 593-600.

[27] D. Vasisht, A. Damianou, M. Varma, and A. Kapoor, "Active learning for sparse bayesian multilabel classification," in KDD, 2014, pp. 472-481.

[28] B. Yang, J.-T. Sun, T. Wang, and Z. Chen, "Effective multi-label active learning for text classification," in $K D D, 2009$, pp. 917-926. 
[29] N. Roy and A. Mccallum, "Toward optimal active learning through sampling estimation of error reduction," in ICML, 2001, pp. 441-448.

[30] X. Li, L. Wang, and E. Sung, "Multilabel svm active learning for image classification," in ICIP, 2004, pp. 2207-2210.

[31] R. Chattopadhyay, W. Zheng, F. Wei, I. Davidson, S. Panchanathan, and J. Ye, "Batch mode active sampling based on marginal probability distribution matching," TKDD, vol. 7, no. 3, pp. 741-749, 2012.

[32] S. Dasgupta and D. Hsu, "Hierarchical sampling for active learning," in ICML, 2008, pp. 208-215.

[33] J. Zhu, H. Wang, B. K. Tsou, and M. Ma, "Active learning with sampling by uncertainty and density for data annotations," TASLP, vol. 18 , no. 6 , pp. 1323-1331, 2010.

[34] B. Du, Z. Wang, L. Zhang, L. Zhang, and D. Tao, "Robust and discriminative labeling for multi-label active learning based on maximum correntropy criterion," TIP, vol. 26, no. 4, pp. 1694-1707, 2017.

[35] Y.-P. Tang and S.-J. Huang, "Self-paced active learning: Query the right thing at the right time," in AAAI, 2019, pp. 5117-5124.

[36] Z. Wang and J. Ye, "Querying discriminative and representative samples for batch mode active learning," in TKDD, vol. 9, no. 3, 2015, pp. 1-23.

[37] E. Elhamifar, G. Sapiro, A. Yang, and S. Shankar Sasrty, "A convex optimization framework for active learning," in ICCV, 2013, pp. 209-216.

[38] A. Kapoor, R. Viswanathan, and P. Jain, "Multilabel classification using bayesian compressed sensing," in NeurIPS, 2012, pp. 2645-2653.

[39] P. Donmez and J. G. Carbonell, "Proactive learning: cost-sensitive active learning with multiple imperfect oracles," in CIKM, 2008, pp. 619-628.

[40] G. Yu, J. Tu, J. Wang, C. Domeniconi, and X. Zhang, "Active multi-label crowd consensus," TNNLS, vol. 99, no. 1, pp. 1-12, 2020.

[41] S.-Y. Li, Y. Jiang, N. V. Chawla, and Z.-H. Zhou, "Multi-label learning from crowds," TKDE, vol. 31, no. 7, pp. 1369-1382, 2019.

[42] M. P. Kumar, B. Packer, and D. Koller, "Self-paced learning for latent variable models," in NeurIPS, 2010, pp. 1189-1197.

[43] L. Lin, K. Wang, D. Meng, W. Zuo, and L. Zhang, "Active self-paced learning for cost-effective and progressive face identification," TPAMI, vol. 40, no. 1, pp. 7-19, 2017.

[44] S.-J. Huang, S. Chen, and Z.-H. Zhou, "Multi-label active learning: Query type matters," in IJCAI, 2015, pp. 946-952.

[45] T. G. Dietterich, R. H. Lathrop, and T. Lozano-Pérez, "Solving the multiple instance problem with axis-parallel rectangles," Artificial Intelligence, vol. 89, no. 1-2, pp. 31-71, 1997.

[46] Z.-H. Zhou, M.-L. Zhang, S.-J. Huang, and Y.-F. Li, "Multi-instance multilabel learning," Artificial Intelligence, vol. 176, no. 1, pp. 2291-2320, 2012.

[47] B. Settles, M. Craven, and S. Ray, "Multiple-instance active learning," in NeurIPS, 2008, pp. 1289-1296.

[48] J. Meessen, X. Desurmont, J.-F. Delaigle, C. De Vleeschouwer, and B. Macq, "Progressive learning for interactive surveillance scenes retrieval," in CVPR, 2007, pp. 1-8.

[49] D. Zhang, F. Wang, Z. Shi, and C. Zhang, "Interactive localized content based image retrieval with multiple-instance active learning," Pattern Recognition, vol. 43, no. 2, pp. 478-484, 2010.

[50] J. Melendez, B. van Ginneken, P. Maduskar, R. H. Philipsen, H. Ayles, and C. I. Sánchez, "On combining multiple-instance learning and active learning for computer-aided detection of tuberculosis," $T M I$, vol. 35 , no. 4, pp. 1013-1024, 2016.

[51] M.-A. Carbonneau, V. Cheplygina, E. Granger, and G. Gagnon, "Multiple instance learning: A survey of problem characteristics and applications," Pattern Recognition, vol. 77, pp. 329-353, 2018.

[52] S. Andrews, I. Tsochantaridis, and T. Hofmann, "Support vector machines for multiple-instance learning," in NuerIPS, 2003, pp. 577-584.

[53] Q. Tan, Y. Yu, G. Yu, and J. Wang, "Semi-supervised multi-label classification using incomplete label information," Neurocomputing, vol. 260, pp. 192-202, 2017.

[54] F. Briggs, X. Z. Fern, and R. Raich, "Rank-loss support instance machines for miml instance annotation," in $K D D, 2012$, pp. 534-542.

[55] M.-L. Zhang and Z.-H. Zhou, "Ml-knn: A lazy learning approach to multi-label learning," Pattern Recognition, vol. 40, no. 7, pp. 2038-2048, 2007.

[56] M. R. Boutell, J. Luo, X. Shen, and C. M. Brown, "Learning multi-label scene classification," Pattern Recognition, vol. 37, no. 9, pp. 1757-1771, 2004.

[57] J. Winn, A. Criminisi, and T. Minka, "Object categorization by learned universal visual dictionary," in ICCV, 2005, pp. 1800-1807.

[58] Y. Chen, J. Bi, and J. Z. Wang, "Miles: Multiple-instance learning via embedded instance selection," TPAMI, vol. 28, no. 12, pp. 1931-1947, 2006.

[59] O. Maron and T. Lozano-Pérez, "A framework for multiple-instance learning," in NeurIPS, 1998, pp. 570-576.
[60] C. C. Chang and C. J. Lin, "Libsvm: a library for support vector machines," TIST, vol. 2, no. 3, p. 27, 2011

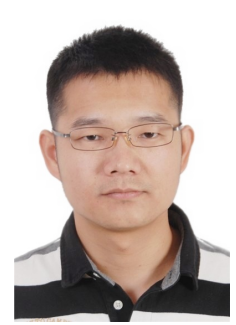

Guoxian Yu is a Professor at the School of Software, Shandong University, Jinan, China. He received the Ph.D. in Computer Science from South China University of Technology, Guangzhou, China in 2013. His current research interests include data mining and bioinformatics. He serves as reviewers for TKDE, TNNLS, TCYB, TCBB, KDD, ICDM, IJCAI and other prestigious journals and conferences.

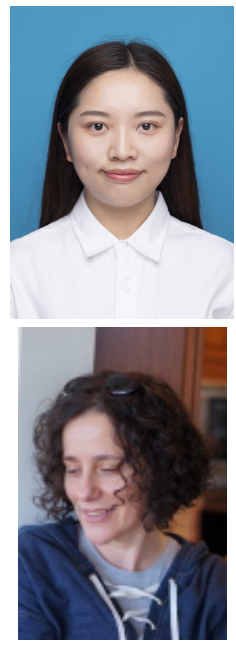

Xia Chen received the M.Sc. degree from the College of Computer and Information Science, Southwest University, Chongqing, China in 2019. She is currently an algorithm engineer with the Alibaba Group, specializing in abnormal community identification. Her current research interests include community detection, anomaly detection, causal inference.

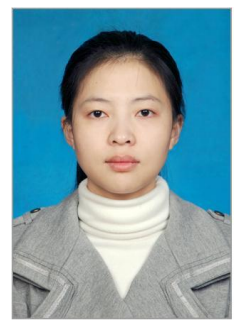

Carlotta Domeniconi is an Associate Professor in the Department of Computer Science at George Mason University since August 2008. Her research interests include machine learning and data mining, with applications in text mining and bioinformatics. She has published extensively in premier journals and conferences in machine learning and data mining. She has served as PC member for KDD, ICDM and AAAI, and she is an Associate Editor of TKDE and KAIS.

Jun Wang is a Professor at the Joint SDU-NTU Centre for Artificial Intelligence Research, Shandong University, Jinan, China. She received B.Sc. degree in Computer Science, M.Eng. degree in Computer Science and Ph.D. in Artificial Intelligence from Harbin Institute of Technology, Harbin, China in 2004, 2006 and 2010, respectively. Her current research interests include machine learning, data mining and their applications in bioinformatics.

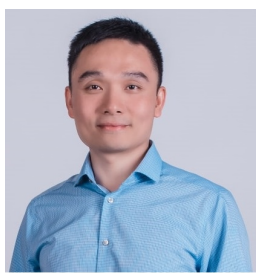

Zhao Li received the Ph.D. degree (Hons.) from the Computer Science Department, University of Vermont. $\mathrm{He}$ is currently a Senior Staff Scientist with the Alibaba Group, specializing in ecommerce ranking and recommendation systems. He has published more than 50 articles in prestigious conferences and journals. His current research interests include adversarial machine learning, network representation learning, knowledge graphs, multi-agent reinforcement learning, and big datadriven security.

Zili Zhang is a Professor at the College of Computer and Information Science, Southwest University, Chongqing, China, and a senior lecturer at Deakin University. His current research interests include bioinspired artificial intelligence, agentbased computing, big data analysis, and agentdata mining interaction and integration.
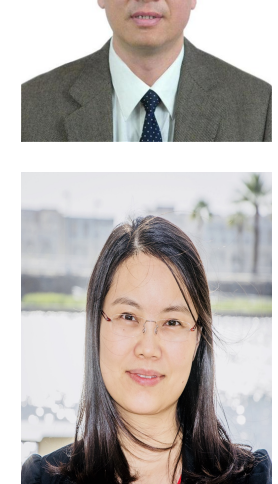

Xiangliang Zhang is an Associate Professor and directs the Machine Intelligence and Knowledge Engineering (MINE) Laboratory in King Abdullah University of Science and Technology (KAUST). She earned her PhD degree in Computer Science with great honors from INRIA-University ParisSud 11, France, in 2010. Her main research interests and experiences are in diverse areas of machine learning and data mining. 